\title{
Protein acylation by saturated very long chain fatty acids and endocytosis are involved in necroptosis
}

Apoorva Pradhan ${ }^{1, \ddagger}$, Daniel Lu ${ }^{1, \ddagger}$, Laura R. Parisi ${ }^{1}$, Shichen Shen ${ }^{2}$, llyas A. Berhane ${ }^{1}$, Samuel L. Galster ${ }^{1}$, Kiana Bynum ${ }^{3}$, Viviana Monje-Galvan ${ }^{4}$, Omer Gokcumen ${ }^{5}$, Sherry R. Chemler ${ }^{1}$, Jun $\mathrm{Qu}^{2}$, Jason Kay ${ }^{3}$, G. Ekin Atilla-Gokcumen ${ }^{1^{*}}$

${ }^{1}$ Department of Chemistry, University at Buffalo, The State University of New York, Buffalo, New York 14260, United States

${ }^{2}$ Department of Pharmaceutical Sciences, University at Buffalo, The State University of New York, Buffalo, New York 14214, United States

${ }^{3}$ Department of Oral Biology, University at Buffalo, The State University of New York, Buffalo, New York 14214, United States

${ }^{4}$ Department of Chemical and Biological Engineering, University at Buffalo, The State University of New York, Buffalo, New York 14260, United States

${ }^{5}$ Department of Biological Sciences, University at Buffalo, The State University of New York, Buffalo, New York, 14260, United States

¥These authors contributed equally

*Lead Contact 


\section{SUMMARY}

Necroptosis is a form of regulated cell death that is characterized by membrane permeabilization. This permeabilization is responsible for the inflammatory properties of necroptosis and is critical for disease states involving this process. We previously showed that very long chain fatty acids (VLCFAs) are functionally involved in necroptosis, potentially through protein fatty acylation. Here, we define the scope of protein acylation by saturated VLCFAs during necroptosis. We show that mixed lineage kinase like protein (MLKL) and phosphoMLKL, key proteins for membrane permeabilization, are exclusively acylated during necroptosis. Reducing the levels of VLCFAs decreases their membrane recruitment, suggesting that acylation by VLCFAs contributes to their membrane localization. Acylation of phosphoMLKL occurs downstream of phosphorylation and oligomerization and appears to be, in part, mediated by ZDHHC5 (a palmitoyl transferase). We also show that disruption of the clathrin-mediated endocytosis increases cell viability during necroptosis, likely by removing phosphoMLKL from the plasma membrane. 


\section{INTRODUCTION}

Programmed cell death is a critical component for maintaining cellular homeostasis. Imbalance between cell proliferation and cell death is linked to many phenotypes, including cancer formation, neurodegenerative disorders and inflammation ${ }^{1}$. Multiple forms of regulated cell death that are executed by distinct pathways have recently been characterized. A fundamental phenotypic difference between them and apoptosis is the permeabilization of the cellular membranes early on in the process which causes an inflammatory phenotype. ${ }^{2}$

Necroptosis is one of these inflammatory programmed cell death pathways and is dependent on the activity of receptor interacting protein kinase 1, 3 (RIPK1, RIPK3) and mixed lineage kinase like protein (MLKL) phosphorylation when caspase activity is reduced. ${ }^{3-4}$ Necroptosis exhibits phenotypic hallmarks of necrosis including cellular swelling, loss of plasma membrane integrity, and the release of intracellular content. ${ }^{3-4}$ Closely linked to this membrane permeabilization and associated inflammatory response, necroptotic cell death has been observed in many diseases, including ischemia/reperfusion injuries ${ }^{5}$ and myocardial infarction ${ }^{6}$.

MLKL oligomerization and translocation to the plasma membrane is required for necroptosis ${ }^{7}$. MLKL consists of a C-terminal kinase-like domain that interacts with RIPK3, which results in its phosphorylation ${ }^{8}$. This phosphorylation triggers the oligomerization and translocation of pMLKL to the plasma membrane. ${ }^{9}$ The membrane recruitment of the oligomer is mediated by electrostatic interactions between positively charged regions of pMLKL and negatively charged phosphatidylinositol phosphate-rich domains of the membrane. ${ }^{10}$ While it seems clear from a number of studies that the membrane association of pMLKL oligomers are important for membrane permeabilization ${ }^{7,11-12}$, the mechanism of membrane permeabilization induced by pMLKL oligomers is not completely understood. It is unclear how pMLKL oligomers impact membrane packing, whether other chemical transformations that pMLKL undergo impact membrane association and the presence of the oligomers induces further phosphatidylinositol recruitment to the protein binding site.

In parallel to its plasma membrane recruitment via canonical RIPK3/RIPK1 activity during necroptosis, vesicular trafficking of MLKL plays a critical role in its cellular localization. MLKL can mediate endosomal trafficking involving constitutive generation of vesicles that can fuse with lysosomes or the plasma membrane irrespective of phosphorylation by RIPK3 and this phenomenon is further enhanced after phosphorylation. ${ }^{13}$ Furthermore, trafficking that results in 
the removal of pMLKL from the plasma membrane may lead to delay in membrane permeabilization and cell death during necroptosis. ${ }^{13}$

Motivated by lipids' role in numerous membrane-related transformations, our laboratory has investigated the role of lipids in necroptosis, specifically during membrane permeabilization. We found that saturated very long chain fatty acids (VLCFAs) accumulated during necroptosis via activation of fatty acid biosynthesis and elongation. ${ }^{14}$ We also showed that VLCFAs have a higher propensity to disturb membrane packing in liposomes and in cells most likely due to interleaflet interdigitation. ${ }^{15}$ One intriguing observation was that in liposomes and during molecular dynamics simulations, high concentrations of VLCFAs were required to induce membrane permeabilizations whereas the intracellular concentration required for plasma membrane permeabilization was much lower. These observations suggest that VLCFAs can achieve high local membrane concentrations in cells during necroptosis. We envisioned that one way in which VLCFAs can reach high local membrane concentration was through their incorporation to proteins and the recruitment of the protein-lipid complex to distinct membrane locales. Since fatty acylation by saturated VLCFAs is understudied, we utilized $\omega$-alkynyl VLCFAs to investigate the covalent modification of proteins with VLCFA in both control and necroptotic cells and found that certain proteins may exhibit increased labeling with VLCFAs during necroptosis. ${ }^{15}$

In this work, we identify the proteins that are acylated by VLCFAs using a clickable fatty acid analog and investigated the involvement of acylation by VLCFAs in necroptosis. We first carried out quantitative proteomics with a unique lonStar approach ${ }^{16}$ to identify proteins that are differentially fatty acylated during this process. We observed that there was an overall decrease in proteins that are acylated by VLCFAs (referred to as VLCFAcylated here on) during necroptosis. However, importantly, MLKL and $\mathrm{pMLKL}$ were among the proteins that were VLCFAcylated in necroptotic cells, but not in control cells. Enrichment analysis highlighted the involvement of the endocytic/lysosomal pathway during necroptosis. Perturbing the endocytic trafficking using a small molecule inhibitor resulted in increased cell viability and decreased the levels of membrane localized pMLKL suggesting the removal of pMLKL from the membrane via exocytosis or degradation. Our results show for the first time the differential protein fatty acylation by a VLCFA during necroptosis and suggests a novel mechanism of membrane recruitment and maintenance of $\mathrm{pMLKL}$ in this process.

\section{RESULTS}


Differential incorporation of $\omega$-alkynyl C20:0 fatty acid during necroptosis. Our previous studies on establishing the changes in the lipid landscape during necroptosis showed that saturated VLCFAs are strongly upregulated during this process ${ }^{14}$ and are involved in membrane permeabilization ${ }^{15}$. We have also shown that these VLCFAs could be incorporated into proteins and this fatty acylation might play a role in necroptosis, for instance by targeting of VLCFAs to certain membrane regions resulting in high local concentrations, mediated by the proteinmembrane interactions. ${ }^{15}$ Here, in order to investigate the role of fatty acylation by VLCFAs and identify the proteins modified by VLCFAs during necroptosis, we analyzed the proteins that are modified by a representative VLCFA during this process using quantitative proteomics. We induced necroptosis in HT-29 human colorectal adenocarcinoma epithelial cells with BV6/zVADFMK/TNF- $\alpha$ treatment, confirmed by the decrease in cell viability (Figure 1A) and membrane localization of pMLKL (Figure 1B, Figure S1A). Following that, we used $\omega$-alkynyl C20:0 fatty acid (C20 alkFA) to identify proteins that are modified with this probe using delivery and enrichment protocols we described previously ${ }^{15}$ (Figure 1C, $n=3$ for each condition, see Materials and Methods). After collecting cell pellets and isolating membrane fraction by ultracentrifugation, we installed a biotin reporter by copper catalyzed alkyne-azide cycloaddition (CUAAC) and captured proteins that are covalently modified by C20 alkFA (referred to as enriched portion from here on). We then carried out quantitative proteomics in order to identify proteins that are acylated by C20 alkFA and compared their levels in control and necroptotic cells. Briefly, once the proteins were enriched on the neutravidin beads, beads were resuspended in detergent containing buffers and were then subjected to a surfactant-aided precipitation/on-bead digestion procedure modified from a recently published method ${ }^{17}$ (see Materials and Methods for details, Figure 1C). Derived tryptic peptides were analyzed by a welloptimized trapping nano LC-Orbitrap mass spectrometry system. Each sample was analyzed twice, once by Orbitrap (OT) and once by Ion Trap (OT) to allow accurate and sensitive peptide detection as well as cross validation of protein identification results. Proteomic quantification was accomplished by lonStar, an in-house developed MS1 ion current-based quantitative proteomics method (Figure 1C, see Materials and methods for details) ${ }^{16}$. As a result, a total of 1672 proteins were quantified with high precision and no missing data across samples in the same condition.

The scatter plots in Figure S1 show the correlation between the mean intensities (calculated by summing up the area under the curve of all peptides inferred to a protein) of different biological replicates of each protein detected in control and necroptotic cells (Figure S1B). Based on the strong correlation between OT and IT dataset $\left(R^{2}>0.99\right.$ for control and 
necroptotic samples), we concluded that our measurements reflect biological rather than technical variation.

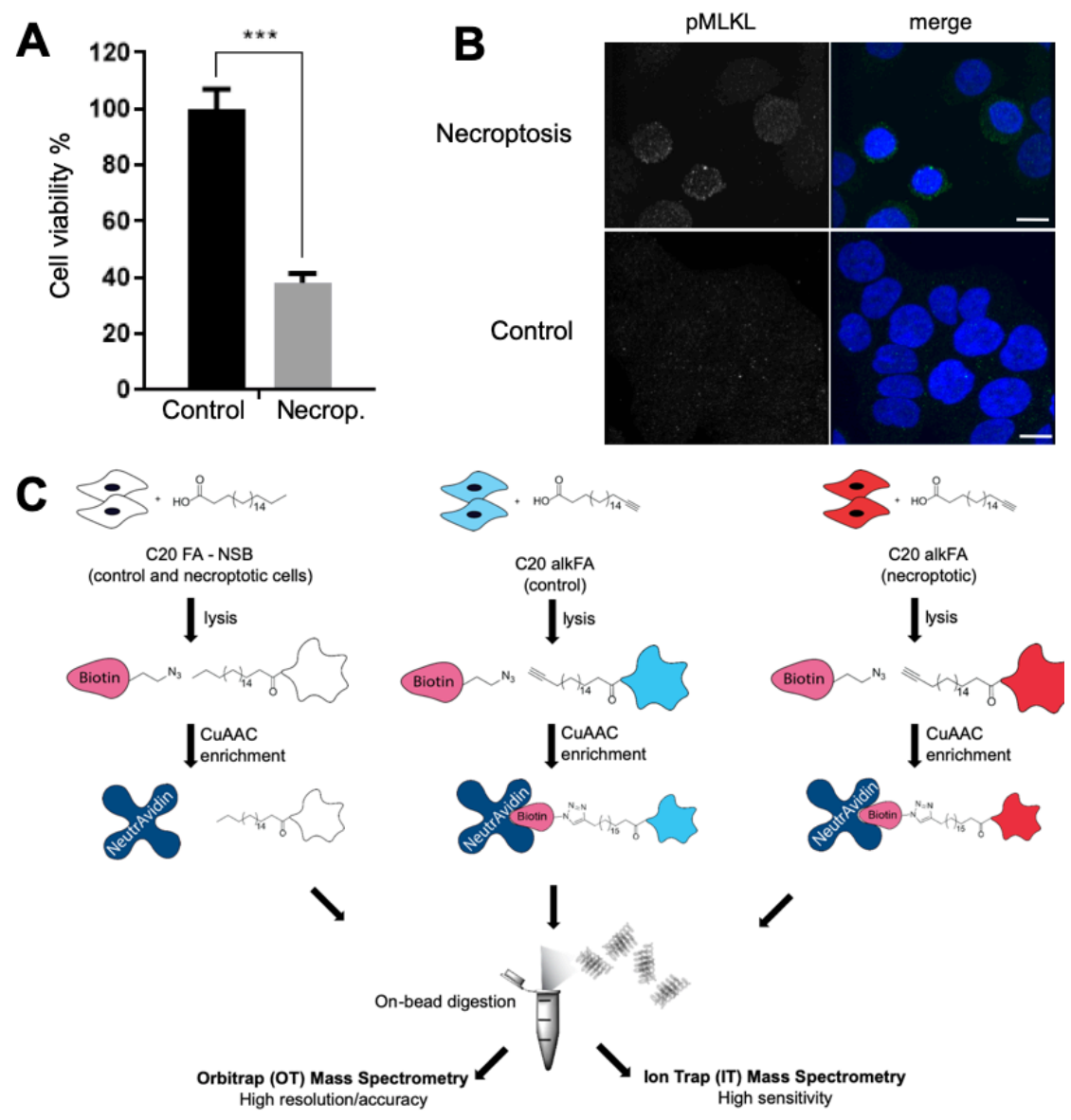

Figure 1. Induction of necroptosis and workflow of C20 fatty acid acylation and proteomics. (A) Cell viability decreases as HT-29 cells undergo necroptosis. Necroptosis is induced with BV6/zVAD-FMK/TNF- $\alpha$ treatment. Error bars represent $1 \mathrm{SD} ; \mathrm{n}=5$. ${ }^{* * *}$ represents $p$ $<0.001$. (B) Cellular distribution of pMLKL during necroptosis. Sample 3D project images of pMLKL immunostaining in necroptotic (top) or control (bottom) cells show an increase in pMLKL staining, mainly concentrated at the plasma membrane. Merge images include DAPI nuclear staining (blue) and pMLKL (green). Scalebar represents $10 \mu \mathrm{m}$. (C) HT-29 cells were treated with endogenous C20:0 FA or C20:0 alkFA for 3h to account for non-specific and specific interactions respectively. Control and necroptotic cells were then membrane fractionated, subjected to CuAAC and installed with a biotin reporter. Biotinylated proteins were captured on neutravidin and subjected to on-bead trypsin digestion followed by quantitative proteomics.

In order to eliminate proteins that are enriched due to non-specific interactions between the proteins and neutravidin resin, we treated cells with endogenous C20:0 FA and subjected them to the same enrichment and analysis protocols (Figure 1C, C20 FA-NSB). We excluded any proteins that we identified in these non-specific binding samples as they should not interact 
with neutravidin resin due to the lack of biotin modification. Out of the total 1672 proteins that were quantified in samples enriched from control and necroptotic cells, 1267 were detected with higher abundance in control cells and 405 were detected with higher abundance in necroptotic condition (Table S1). Figure 2A shows the difference in mean intensities of proteins detected (total of 1672 proteins) in control and necroptotic cells in OT (x axis) and IT (y axis) platforms. Consistent with the high correlations we report in Figure $S 1$ for the intensities detected in these platforms, we also observe a strong correlation between the differences in mean intensities for detected proteins in control and necroptotic conditions showing that these differences reflect biological variation rather than technical ones. Furthermore, an interesting observation was that a large number of proteins appeared to be only acylated in control (561, dark blue in Figure 2B) or in necroptotic (158, dark red in Figure 2B) cells (Table S1). Based on these results, it is clear that overall there are fewer proteins acylated by VLCFA during necroptosis (Figure 2B, Figure S2), however there is a distinct group of 158 proteins that are VLCFAcylated exclusively in necroptotic cells.
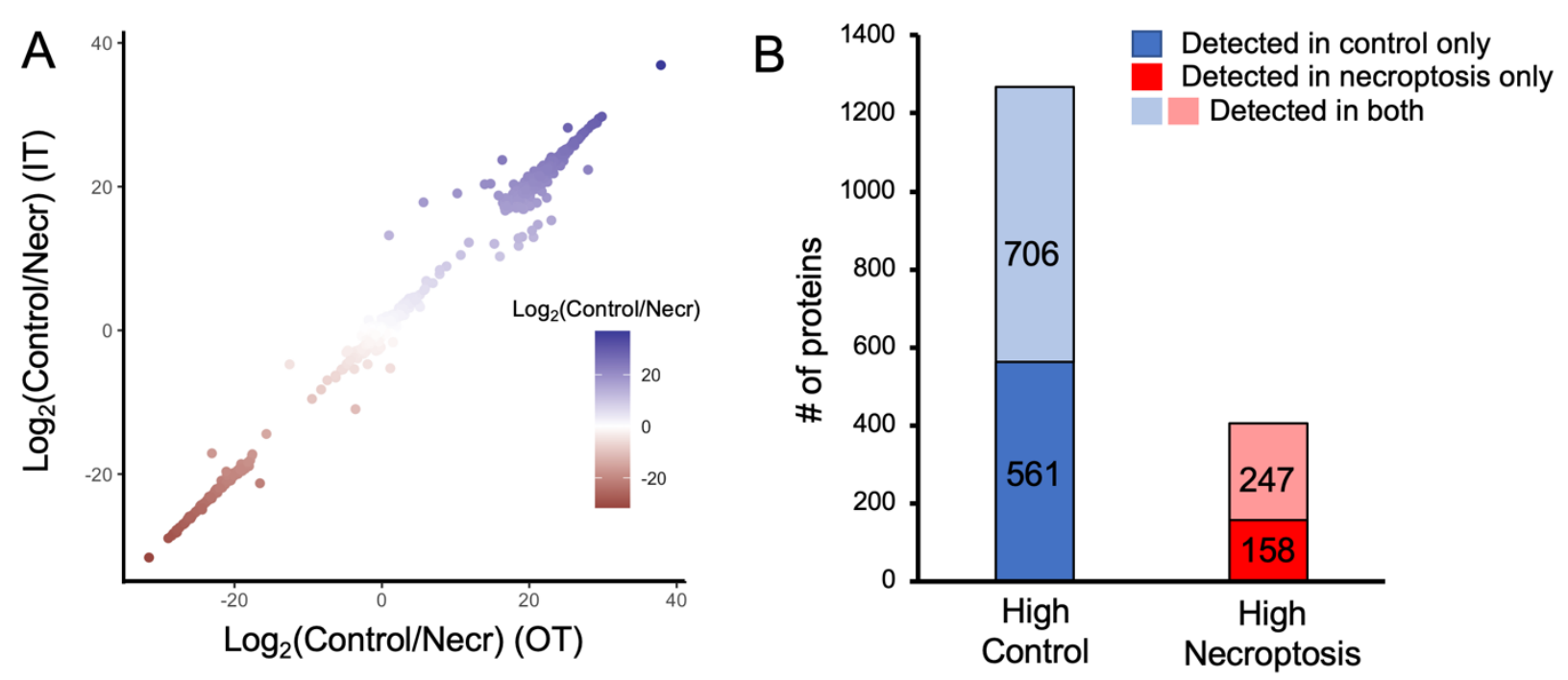

Figure 2. Quantitative analysis of acylated proteome in necroptosis. (A) High correlation between the mean intensities of acylated proteins detected in control and necroptotic cells in Orbitrap Lumos (x axis) and lon trap (y axis) platforms. Strong correlation between difference in mean intensities indicates biological variation rather than technical variation among the proteins detected in control and necroptotic cells. (B) Bar plot shows the total number of proteins (1672) undergoing acylation in either control, necroptotic or both conditions. Blue and red bars represent the number of proteins with high abundance in control or necroptotic cells, respectively. A higher number of proteins appear acylated in only control (561) as compared to only necroptosis (158). Overall, fewer proteins undergo acylation in necroptosis (405) as compared to control (1267). 
MLKL and pMLKL are acylated during necroptosis. A total of 1332 proteins showed differential acylation by VLCFAs significantly $(p<0.01)$ during necroptosis: the acylation of 285 proteins were upregulated during necroptosis whereas 1047 showed a reduction in VLCFAcylation in the process (Table S1). Among the 285 proteins with increased VLCFAcylation during necroptosis, 158 were only detected in necroptotic cells. Based on our previous work suggesting increased VLCFA levels and protein fatty acylation could be involved in necroptosis ${ }^{14-15}$, we initially turned our attention to proteins with increased VLCFAcylation during necroptosis.

Intriguingly, MLKL was one of these proteins which appeared to be modified by C20:0 alkFA only during necroptosis. Our proteomics data set is limited in distinguishing peptides from unmodified ones, therefore little information was obtained whether MLKL and/or PMLKL was acylated. Hence, we investigated the fatty acylation of MLKL and pMLKL using western blotting. We enriched fatty acylated proteins from control and necroptotic cells as we described above and analyzed protein content using western blotting. We detected both MLKL and pMLKL in necroptotic cells but not in control cells suggesting that both MLKL and PMLKL can be fatty acylated during necroptosis and that their acylation is not appreciable in the absence of necroptotic activity (Figure 3A). In order to understand the nature of acylation, we treated control and necroptotic cells with C20:0 alkFA, fractionated membrane proteins and installed Biotin-azide. We then treated the lysates with hydroxylamine $\left(\mathrm{NH}_{2} \mathrm{OH}\right)$ which cleaves thioester linkages ${ }^{18}$, and carried out protein enrichment through neutravidin beads. When we subjected the bead-bound proteins to western blotting, MLKL and pMLKL signal from necroptotic samples completely disappeared with $\mathrm{NH}_{2} \mathrm{OH}$ treatment compared to control samples, suggesting that MLKL and pMLKL may be acylated via S-linkage (Figure 3B). 

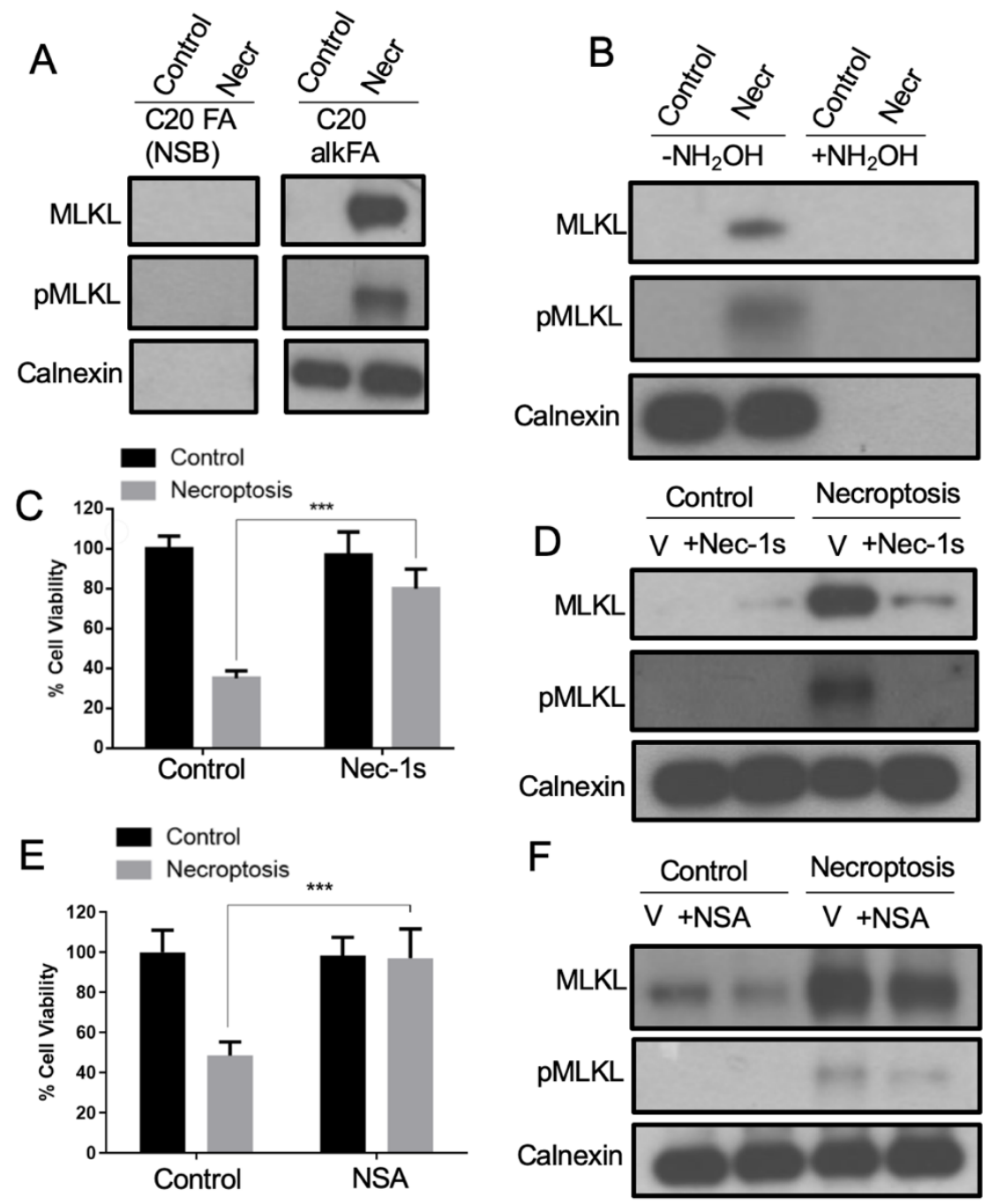

Figure 3. MLKL and pMLKL are acylated downstream of RIPK1 activation. (A) Western blot analysis of proteins from $\mathrm{C} 20$ alkFA treated control and necroptosis cells. After cell lysis and Biotin-azide attachment via CuAAC, acylated proteins are enriched on neutravidin resin. The samples are blotted for MLKL and PMLKL and Calnexin. Calnexin is used as a loading control. We note that the treatments with C20 FA and C20 alkFA and the western blot analysis were carried out during the same experiment. Uncropped western blot image in shown in Figure S3B. (B) Acylation of MLKL and pMLKL with C20 alkFA show hydroxylamine sensitivity. Control and necroptotic cells treated C20 alkFA were membrane fractionated and subjected to CuAAC with Biotin-azide. The proteins were treated with $0.3 \mathrm{M}$ hydroxylamine for $1 \mathrm{~h}$, enriched using neutravidin beads, then blotted for MLKL and pMLKL and Calnexin. Calnexin is used as a positive control for hydroxylamine sensitivity. We note that $\mathrm{NH}_{2} \mathrm{OH}$ treatment does not cause protein degradation (data not shown). Reduction of signal with hydroxylamine treatment indicates that MLKL and pMLKL acylation occurs primarily through S-linkages. (C and E) $1 \mu \mathrm{M}$ $\mathrm{Nec}-1 \mathrm{~s}(\mathbf{C})$ and $1 \mu \mathrm{M}$ NSA (E) reduce necroptotic cell death. Percent cell viability of control, $\mathrm{Nec}-1 \mathrm{~s}$ or NSA-treated and necroptotic cells are shown. Necroptosis is induced with BV6/zVADFMK/TNF- $\alpha$ treatment. Error bars represent 1 SD; $n=5$. ${ }^{* * *}$ represents $p<0.001$. (D and F) Western blot analysis of C20 alkFA treated control and necroptotic cells in the presence of Nec$1 \mathrm{~s}$ and NSA. Control and necroptotic cells treated with C20 alkFA and Nec-1s/NSA were membrane fractionated and subjected to CuAAC with Biotin-azide and enrichment using 
neutravidin beads. The proteins on the beads were blotted for MLKL and pMLKL. Nec-1s (D) and NSA (F) treatment reduce VLCFAcylation of MLKL and pMLKL.

\section{pMLKL acylation occurs downstream of RIPK1 activity and its membrane localization.} RIPK1 activity is critical for MLKL phosphorylation and its translocation to plasma membrane during necroptosis. ${ }^{7}$ In order to investigate whether the acylation of pMLKL occurs downstream of RIPK1 activation during necroptosis, we used a small molecule inhibitor of RIPK1, Necrostatin-1s (Nec-1s), which increased cell viability in the presence of necroptotic activity (Figure 3C). We treated necroptotic cells and Nec-1s-protected cells with C20 alkFA, fractionated membrane proteins and installed Biotin azide. We then enriched for proteins that are modified with C20 alkFA using neutravidin as described above. Figure 3D shows the levels of MLKL and pMLKL in the enriched portion in necroptotic and Nec-1s-protected cells compared to control cells. We detected very low MLKL and $\mathrm{pMLKL}$ in the protected group, suggesting that no appreciable fatty acylation of pMLKL takes place in protected cells. This finding is important because it suggests that pMLKL is acylated downstream of RIPK1 activity (Figure 3D).

The next step in canonical necroptosis signaling after MLKL phosphorylation is the oligomerization of pMLKL, membrane recruitment, and binding. Using a similar approach as we describe above for inhibiting RIPK1 activity, we used a small molecule necrosulfoamide ${ }^{8}$ (NSA) which prevents the oligomerization of $\mathrm{pMLKL}$, membrane translocation and rescues cell death in necroptosis (Figure 3E). We treated necroptotic cells and NSA-protected cells with C20 alkFA and enriched for proteins that are acylated with C20 alkFA using neutravidin. Figure 3F shows the levels of acylated MLKL and pMLKL in necroptotic and NSA-protected cells compared to control cells. We detected a significant decrease in acylation of MLKL and PMLKL in the protected group (Figure 3F) whereas their cellular levels did not change (as detected in whole lysates, Figure S3A), suggesting that the acylation of pMLKL occurs downstream to its oligomerization.

\section{ELOVL7 knockdown reduces the membrane recruitment of pMLKL during necroptosis.}

Our results on the acylation of MLKL and PMLKL are based on exogenous delivery of fatty acid of clickable fatty acid analogs. We wanted to confirm that VLCFAcylation occurs in cells and during necroptosis without perturbing lipid levels by exogenous delivery. To achieve this, we tested whether the reduction of VLCFAs would impact the membrane recruitment of pMLKL during necroptosis. If our hypothesis that VLCFAcylation of PMLKL is functionally important for cell death during necroptosis is correct, we should see a decrease in PMLKL acylation (hence 
its membrane recruitment) and a rescue in cell death when the levels of VLCFAs are depleted. We reduced the levels of VLCFAs by lentivirus-based ELOVL7 knockdown ${ }^{15}$ and tested the membrane recruitment of MLKL and pMLKL. The knockdown of ELOVL7 increased the viability of necroptotic cells as expected (Figure 4A) and reduced the levels of membrane-associated pMLKL during necroptosis (Figure 4B) supporting that VLCFAcylation of MLKL and pMLKL could be important for necroptotic cell death.
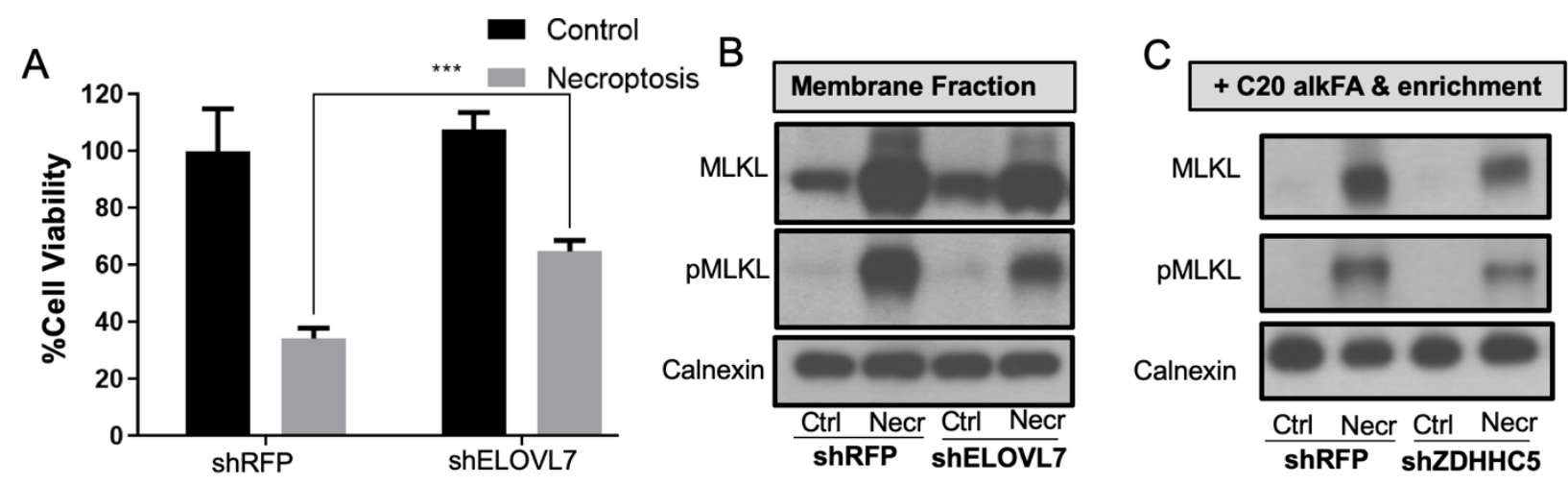

Figure 4. Knockdown of ELOVL7 and ZDHHC5 reduces cell death and membrane recruitment of MLKL and pMLKL during necroptosis. (A) ELOVL7 knockdown reduces necroptotic cell death. Percent cell viability of shRFP and shELOVL7 transduced cells treated with BV6/zVAD-FMK/TNF-alpha to induce necroptosis compared to vehicle-treated control cells. Error bars represent $1 \mathrm{SD} ; \mathrm{n}=5$. ${ }^{* * *}$ represents $p<0.001$ (B) Western blot analysis of membrane fraction from untreated and necroptotic shRFP and shELOVL7 cells. Cell lysates are fractionated by ultracentrifugation and membrane fractions are analyzed. Decrease in MLKL and pMLKL are observed in necroptotic shELOVL7 as compared to necroptotic control cells suggesting that membrane recruitment is reduced in shELOVL7 cells. (C) Control and necroptotic shRFP and shZDHHC5 cells treated with C20 alkFA were membrane fractionated and subjected to CuAAC with Biotin-azide and enrichment using neutravidin beads. The proteins on the beads were blotted for MLKL, pMLKL and calnexin. $\sim 40 \%$ decrease in the enriched pMLKL is observed as compared to necroptotic control cells suggesting role of ZDHHC5 in VLCFAcylation during necroptosis.

ZDHHC5 is in part responsible for MLKL acylation. Our results suggest that the incorporation of VLCFAs to MLKL and pMLKL are S-linked. ${ }^{14}$ S-linked acylation is a posttranslational modification where fatty acids are covalently attached onto proteins via thioester bond. ${ }^{19}$ These modifications are catalyzed by a family of 23 protein acyltransferases (ZDHHCs). All proteins of this family have a conserved catalytic zinc binding site, aspartic acid-histidinehistidine-cysteine (DHHC) cytosolic domain and several transmembrane domains. ${ }^{19-20}$ The family members share less homology within the $\mathrm{N}$-and $\mathrm{C}$-terminal cytosolic domains which are responsible for their recognition with specific substrates, although redundancy between different 
members have been reported. ${ }^{19,21}$ The substrate specificity of ZDHHCs are also greatly impacted by the cellular (co)localization with substrates at the plasmalemma, ER, Golgi or endosomal membranes. Based on the spatial organization of ZDHHCs, these enzymes acylate a variety of target proteins and regulate their targeting to distinct cellular membranes. ${ }^{19}$

Many members of the ZDHHC S-acyltransferases reside at the endoplasmic reticulum and Golgi apparatus and ZDHHC5 is one of the few members of the family that is localized to the plasma membrane and endosomal compartments. ${ }^{22-23}$ Studies have shown that the acylation of target proteins by $\mathrm{ZDHH} 5$ takes place at the plasma membrane and retains them at this location, controlling their subcellular localization. ${ }^{23-25}$ ZDHHC5 is also involved in regulation of the localization of endocytosis-related proteins that have been linked to MLKL recycling. ${ }^{26}$ Based on these observations and the fact that pMLKL acylation occurs downstream to its oligomerization, potentially at the plasma membrane, we focused on investigating ZDHHC5 as a potential acyltransferase that might be responsible for the incorporation of MLKL/pMLKL. We knocked down ZDHHC5 using lentiviral shRNA (see Methods) and tested whether ZDHHC5 could be responsible for the incorporation of VLCFAs to pMLKL during necroptosis by addition of $\mathrm{C} 20$ alkFA to shRFP and shZDHHC5 cells and subsequent enrichment of proteins containing this probe via click chemistry and using neutravidin beads. We observed a decrease in the levels of enriched pMLKL in shZDHHC5 cells (Figure 4C), suggesting that VLCFAcylation of pMLKL during necroptosis is, at least in part, mediated by ZDHHC5. Overall, our results support that ZDHHC5 activity might play a role in acylation and membrane recruitment and retainment of pMLKL during necroptosis. However, since we still observe VLCFAcylation of pMLKL in shZDHHC5 cells, the involvement of other ZDHHCs in PMLKL acylation is likely.

Enrichment analysis suggests an involvement of endocytosis in necroptosis. Next, in order to gain insights on whether proteins from a specific pathway are targets of VLCFAcylation during necroptosis, we carried out enrichment analysis using the Database for Annotation, Visualization and Integrated Discovery (DAVID) Bioinformatics Resource. ${ }^{27}$ We input proteins that showed differential fatty acylation $(p<0.01)$ during necroptosis into DAVID which then mapped these genes against the KEGG database..$^{28}$ Eight pathways showed significant enrichments ( $p<0.05$, Benjamini Hochberg FDR, Figure 5A, Table S2) in this dataset. Three of the eight pathways (SNARE interactions, lysosome and endocytosis) were vesicular trafficking related (highlighted in gray in Table S2).

Endocytic and lysosomal activity affect MLKL and pMLKL trafficking and recycling from the plasma membrane. The association of pMLKL and MLKL with flotillin 1 and 2 leads to their 
endocytosis followed by lysosomal degradation of pMLKL. ${ }^{13,29}$ Other than endocytosis of MLKL, flotillins were also found to associate with MLKL in exosomes which then results in the extrusion of pMLKL from cells. ${ }^{13,30}$ Studies have also suggested that MLKL and PMLKL associate with proteins of the ESCRT (endosomal sorting complex required for transport) machinery or those accessory to their function which causes a delay in membrane permeabilization during necroptosis. $^{31}$

S-acylation of proteins impacts endocytic activity. ${ }^{32-33}$ Hence based on the differential acylation of endocytic proteins during necroptosis, we hypothesized that endocytic pathway may be involved in necroptosis. To test this hypothesis, we took a pharmacological approach of targeting endocytic pathway using dynasore. Dynasore is a cell permeable and rapidly acting small molecule inhibitor for dynamin 1, dynamin 2 and dynamin-related protein 1, which promote fission and budding of the endocytic vesicles on the plasma membrane into the cytosol. ${ }^{34-35}$ We pre-treated HT-29 cells with dynasore, induced necroptosis via addition of BV6 and Z-VAD-fmk followed by TNF- $\alpha$ treatment for $3 \mathrm{~h}$, and assessed the changes in cell viability in the presence of the small molecules. Dynasore pretreatment prevented cell death significantly $(p<0.001)$ compared to necroptotic cells (Figure 5B). We note that the rescue is specific to necroptosis and is not observed in apoptosis suggesting that it is not the inhibition of canonical TNF- $\alpha$ signaling that mediated the rescue (Figure 5B). We then investigated the effect of dynasore treatment in membrane-localized and overall pMLKL levels in necroptotic cells and observed that dynasore treatment strongly reduced the levels of pMLKL in membranes (Figure $\mathbf{5 C}$ ) and in whole lysate, without affecting MLKL levels (Figure 5D). These results suggest that the rescue we observe in cell death could be due to either blocking the phosphorylation of MLKL or removal of pMLKL from the plasma membrane. Based on the previous studies showing the effect of membrane trafficking in clearing PMLKL containing damaged plasma membrane structures, we believe the decrease in PMLKL levels we observe upon inhibiting endocytosis is due to its removal form the plasma membrane, possibly through exocytocytic scission. 


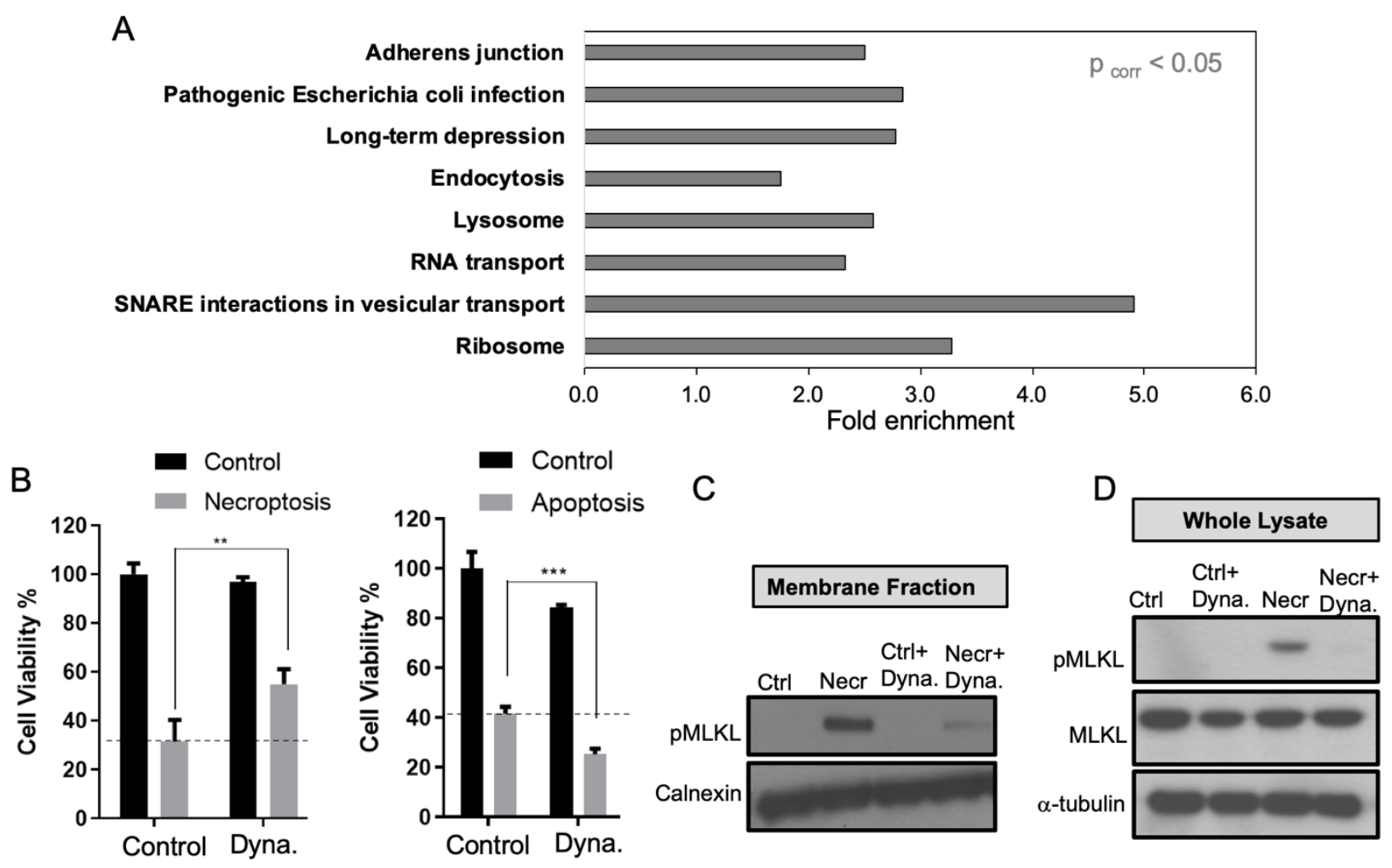

Figure 5. Inhibition of endocytic pathway specifically ameliorate necroptotic cell death. (A) Pathway enrichment analysis of proteins that show differential fatty acylation during necroptosis. 8 pathways significantly enriched (corrected $p$ value $<0.05$ ). (B) Small molecule inhibitor targeting endocytic pathway rescues necroptotic cell death, but not apoptotic cell death, in HT-29 cells. HT-29 cells were pretreated with $100 \mu \mathrm{M}$ dynasore for 2 hours. Vehicle-treated control cells were treated with DSMO. For induction of necroptosis, cells were treated with BV6/zVAD-FMK/TNF- $\alpha$. For induction of apoptosis, cells were treated with BV6/TNF- $\alpha$. The percent cell viability in the presences of inhibitor during necroptosis was compared to the vehicle-treated control cells. Error bars represent $1 \mathrm{SD} ; \mathrm{n} \leq 5 .{ }^{*}$ represents $p<0.05,{ }^{* *}$ represents $p<0.01,{ }^{* * *}$ represents $p<0.001$, ns represents not significant. (C-D) Western blot analysis of membrane fraction (C) and whole lysate (D) of vehicle-treated cells and dynasore treated cells with or without necroptosis. Cell lysates are fractionated by ultracentrifugation and membrane fractions are analyzed. The decrease in pMLKL observed in necroptosis when dynasore is present suggesting endocytosis maybe involved in necroptosis.

\section{DISCUSSIONS/CONCLUSIONS}

There are multiple signaling pathways that can be activated in response to TNF- $\alpha$ activation. This activation, in the presence of low caspase- 8 activity and low activity of the of the cellular inhibitor of apoptosis antagonists, results in the activation of necroptotic signaling, activation of RIPK3, and phosphorylation of MLKL in necroptosomes. ${ }^{36} \mathrm{~A}$ recent study illustrated that downstream to its phosphorylation, MLKL translocates to the plasma membrane via Golgi-, actin- and microtubule-dependent trafficking, a new rate-limiting factor in necroptosis. ${ }^{37}$ Upon 
binding to the plasma membrane, $\mathrm{pMLKL}$ oligomers induce membrane permeabilization ${ }^{36}$ which does not appear to occur via the formation of regularly-structured membrane pores ${ }^{37}$. Based on these observations and others ${ }^{29}$, it is likely that other transformations that pMLKL might undergo contribute to membrane permeabilization or the mechanism that stabilizes pMLKL oligmers at the plasma membrane.

We have previously studied the involvement of lipids in necroptosis and showed that saturated VLCFAs were upregulated via activated biosynthesis and mediate membrane permeabilization. ${ }^{14-15}$ Our results suggested that these VLCFAs might be incorporated into proteins during necroptosis and targeted to certain membrane regions as a result of specific protein-bilayer interactions, allowing high local concentrations of these VLCFAs on the plasma membrane.$^{15}$ In this work, we investigate the role of VLCFAcylation during necroptosis and present two key findings.

First, we show that pMLKL and MLKL are acylated by a representative saturated VLCFA during necroptosis. We have also shown that the acylation of pMLKL occurs downstream to MLKL phosphorylation and plasma membrane localization, suggesting that the acylation takes place at the plasma membrane. MLKL consists of three domains: the 4-helix-bundle which mediates the interactions with the membrane; the brace domain, and the pseudokinase domain that is phosphorylated by RIPK3 ${ }^{38}$ This phosphorylation is critical for membrane recruitment during necroptosis. ${ }^{39}$ Recently, it has been suggested that the 4-helix-bundle also contributes to the membrane recruitment and binding, in addition to the phosphorylation of the pseudokinase domain. ${ }^{40}$ The 4-helix-bundle domain has two predicted sites for palmitoylation (Cys18 and Cys24, SwissPalm ${ }^{41}$ ). Our results suggest that the acylation of $\mathrm{pMLKL}$ and MLKL is S-linked and, in part, mediated by ZDHHC5, a member palmitoyl transferase family, indicating the acylation of 4-helix-bundle domain during necroptosis. The integral ZDHHCs undergo an autoacylation step on the cytosolic DHHC motif. The acylated enzyme intermediate then transfers the acyl group to its substrate. ${ }^{19}$ As one of the few plasma membrane-localized $\mathrm{ZDHHC}$ members, ZDHHC5 mediates the acylation of substrate at the plasma membrane and their plasma membrane recruitment ${ }^{20}$, supporting our findings that of pMLKL and MLKL are acylated at the plasma membrane during necroptosis. Several studies have suggested increased binding of fatty acylated proteins to phosphoinositide lipids. ${ }^{42-43}$ As such, it is possible that the membrane binding of pMLKL is enhanced after its fatty acylation.

It is important to note that similar to what we have observed for modification with C20 alkFA, MLKL and pMLKL can also be acylated by C16 alkFA during necroptosis (Figure S3B). 
However, we believe that modifications by C16 FA and VLCFAs contribute to membrane permeabilization differently during necroptosis. We previously showed that VLCFAs interact with membranes and disrupt their integrity in model liposomes and molecular dynamics simulations. ${ }^{15}$ Based on the modest increases of VLCFAs during necroptosis, we proposed that the incorporation of VLCFAs into proteins can facilitate their recruitment to defined membrane domains and allow high local concentrations of these lipids at the membrane and perturb membrane integrity ${ }^{15}$. Translocation of pMLKL oligomers to the plasma membrane is driven by electrostatic interactions between the oligomer interface and negatively charged phosphatidylinositol phosphate (PIP)-rich membrane domains. ${ }^{44}$ Several PIP species are known to be actively involved in cell signaling cascades, both in endogenous processes as well as in mechanisms of action of viruses and other pathogens. ${ }^{45-47}$ Protein-lipid interactions can alter lipid patterns on the membrane via electrostatic interactions or amino acid insertion into the bilayer. In turn, lateral lipid sorting alters the local mechanical and structural properties of the membrane. ${ }^{48-50}$ Binding of pMLKL oligomers to defined regions of the plasma membrane and further rearrangement of the local lipid distribution at their binding site allows high concentration of fatty acids that covalently modify pMLKL. Acylation of pMLKL oligomers would allow the local recruitment of VLCFAs to membrane regions that already possess different mechanical and structural properties, which facilitates their membrane disruption action.

Second, we define the scope of protein VLCFAcylation during necroptosis using state-ofthe-art quantitative proteomics methods. Overall, we observe a decreased number of proteins are modified by a representative VLCFA during necroptosis. Pathway analysis conducted using proteins that show different levels of VLCFAcylation during necroptosis highlighted the enrichment of proteins belonging to the endocytic and lysosomal pathways. Previous studies have shown that PMLKL can associate with ESCRT proteins and flotillins which is suggested to result in the removal of pMLKL containing regions from the plasma membrane and their degradation. ${ }^{13,30-31}$ In parallel, flotillin association of pMLKL can result in its removal from the plasma membrane via exosomes. Based on these observations, it is clear that vesicular trafficking is key for maintenance of $\mathrm{PMLKL}$ and MLKL at the plasma membrane. We show that inhibition of clathrin-mediated endocytosis resulted in a strong rescue from cell death during necroptosis and decreased the levels of membrane bound PMLKL, whereas the whole cellular MLKL levels remained unchanged, suggesting that the inhibition of endocytosis pathway enhances the removal of pMLKL from the plasma membrane and preserves plasma membrane integrity. These results suggest for the first time that targeting endocytosis could be a way to delay membrane permeability during necroptosis. Overall, our results provide novel findings on 
the role of protein acylation by VLCFAs during necroptosis and endocytic trafficking in maintaining $\mathrm{pMLKL}$ at the plasma membrane during this process.

\section{SIGNIFICANCE}

Necroptosis is a well-characterized form of caspase-independent regulated cell death that is involved in numerous diseases. A hallmark of necroptosis is the membrane permeabilization and rupture that is mediated by mixed linage kinase like protein (MLKL). This membrane rupture is linked to the inflammatory properties of necroptosis and is critical for disease states involving this process. It is established that electrostatic interactions between phosphatidyl inositol phosphates and phosphorylated MLKL (pMLKL) oligomers facilitate the membrane recruitment of pMLKL. In efforts to understand how lipids might contribute to necroptosis, we previously showed that saturated very long chain fatty acids (VLCFAs) accumulate and that they are functionally involved in this process. Our results also indicated that protein fatty acylation by VLCFAs can be a mechanism by which VLCFAs contribute to this process. Here we define the scope of protein acylation by saturated VLCFAs during necroptosis. First we show that, although there is an overall decrease in acylation, some proteins, including MLKL and pMLKL, are exclusively acylated during necroptosis. Reducing the levels of VLCFAs decreases the membrane recruitment of MLKL and PMLKL and rescues from cell death, suggesting that acylation by VLCFAs contributes to membrane localization of pMLKL and subsequent membrane permeabilization. Acylation of MLKL and pMLKL occur downstream of phosphorylation and oligomerization and appear to be S-linked. Second, we show that disruption of clathrin-mediated endocytic pathway results in a strong rescue in cell viability during necroptosis, likely by removal of pMLKL from the plasma membrane. Altogether, we show for the first time that MLKL and pMLKL can be acylated during necroptosis. We also show that disruption of endocytosis presents a promising strategy to delay membrane permeabilization and cell death during necroptosis by promoting the removal of pMLKL from the plasma membrane.

\section{HIGHLIGHTS}

- Saturated VLCFAs are differentially incorporated to proteins during necroptosis.

- $\quad$ PMLKL/MLKL exhibit acylation by VLCFAs in necroptosis.

- Blocking endocytosis results in a strong rescue form cell death during necroptosis. 
eTOC

Pradhan et al. show that MLKL and pMLKL are acylated by saturated very long chain fatty acids during necroptosis and that the disruption of endocytosis decreases the levels of membrane bound PMLKL and MLKL, resulting a rescue from cell death during this process.

\section{EXPERIMENTAL PROCEDURES}

Detailed information on the materials used can be found in the Supplemental Experimental Procedures section, as well as detailed procedures for cell culture and other analyses.

\section{SUPPLEMENTAL INFORMATION}

Supplemental Information includes 3 figures, 2 tables, materials used, experimental procedures and can be found with this article online.

Proteomics data are available via ProteomeXchange with identifier PXD022527

Project Name: Quantitative proteomics characterization of proteins modified by very long chain fatty acids (VLCFAs) under necroptosis

Project accession: PXD022527

\section{AUTHOR CONTRIBUTIONS}

Experiments were designed by A.P., D.L., L.R.P., J.G.K. and G.E.A-G. The experiments were conducted by A.P, D.L., L.R.P. S.S. and J.Q. carried out the proteomics experiments. C20 alkFA synthesis was performed by I.A.B., S.L.G., S.R.C. A.P. and D.L. analyzed the data. Microcopy experiments were conducted by K.B. The manuscript was written by A.P., D.L., J.G.K., and G.E.A-G. The study was directed by G.E.A-G. The authors declare no conflict of interest.

\section{ACKNOWLEDGMENTS}

We acknowledge the support from the National Science Foundation grant (MCB1817468 to G.E.A.G.), the National Institutes of Health (GM078383 to S.R.C.), and the Research Foundation for The State University of New York (J. Solo funds to G.E.A.G.). We thank P. Gollnick (UB Biological Sciences) for ultracentrifuge usage. Microscopy was performed in the Optical Imaging and Analysis Facility (UB Dental Sciences). 


\section{REFERENCES}

1. Thompson, C. B., Apoptosis in the pathogenesis and treatment of disease. Science 1995, 267 (5203), 1456-62.

2. Zhang, Y.; Chen, X.; Gueydan, C.; Han, J., Plasma membrane changes during programmed cell deaths. Cell Res. 2017, Epub Oct 27, 2017. DOI:10.1038/cr.2017.133.

3. Holler, N.; Zaru, R.; Micheau, O.; Thome, M.; Attinger, A.; Valitutti, S.; Bodmer, J. L.; Schneider, P.; Seed, B.; Tschopp, J., Fas triggers an alternative, caspase-8-independent cell death pathway using the kinase RIP as effector molecule. Nat. Immunol. 2000, 1 (6), 489-95. 4. Degterev, A.; Huang, Z.; Boyce, M.; Li, Y.; Jagtap, P.; Mizushima, N.; Cuny, G. D.; Mitchison, T. J.; Moskowitz, M. A.; Yuan, J., Chemical inhibitor of nonapoptotic cell death with therapeutic potential for ischemic brain injury. Nat. Chem. Biol. 2005, 1 (2), 112-119.

5. Lau, A.; Wang, S.; Jiang, J.; Haig, A.; Pavlosky, A.; Linkermann, A.; Zhang, Z. X.; Jevnikar, A. M., RIPK3-mediated necroptosis promotes donor kidney inflammatory injury and reduces allograft survival. American Journal of Transplantation 2013, 13 (11), 2805-18.

6. $\quad$ Luedde, M.; Lutz, M.; Carter, N.; Sosna, J.; Jacoby, C.; Vucur, M.; Gautheron, J.; Roderburg, C.; Borg, N.; Reisinger, F.; Hippe, H. J.; Linkermann, A.; Wolf, M. J.; Rose-John, S.; Lullmann-Rauch, R.; Adam, D.; Flogel, U.; Heikenwalder, M.; Luedde, T.; Frey, N., RIP3, a kinase promoting necroptotic cell death, mediates adverse remodelling after myocardial infarction. Cardiovasc Res 2014, 103 (2), 206-16.

7. Cai, Z.; Jitkaew, S.; Zhao, J.; Chiang, H. C.; Choksi, S.; Liu, J.; Ward, Y.; Wu, L. G.; Liu, Z. G., Plasma membrane translocation of trimerized MLKL protein is required for TNF-induced necroptosis. Nature Cell Biology 2014, 16 (1), 55-65.

8. Sun, L.; Wang, H.; Wang, Z.; He, S.; Chen, S.; Liao, D.; Wang, L.; Yan, J.; Liu, W.; Lei, $X$.; Wang, $X$., Mixed lineage kinase domain-like protein mediates necrosis signaling downstream of RIP3 kinase. Cell 2012, 148 (1-2), 213-227.

9. Wang, H.; Sun, L.; Su, L.; Rizo, J.; Liu, L.; Wang, L.-F.; Wang, F.-S.; Wang, X., Mixed lineage kinase domain-like protein MLKL causes necrotic membrane disruption upon phosphorylation by RIP3. Molecular cell 2014, 54 (1), 133-46.

10. Quarato, G.; Guy, C. S.; Grace, C. R.; Llambi, F.; Nourse, A.; Rodriguez, D. A.; Wakefield, R.; Frase, S.; Moldoveanu, T.; Green, D. R., Sequential Engagement of Distinct MLKL Phosphatidylinositol-Binding Sites Executes Necroptosis. Molecular cell 2016, 61 (4), 589-601.

11. Chen, X.; Li, W.; Ren, J.; Huang, D.; He, W.-T.; Song, Y.; Yang, C.; Li, W.; Zheng, X.; Chen, P.; Han, J., Translocation of mixed lineage kinase domain-like protein to plasma membrane leads to necrotic cell death. Cell research 2014, 24 (1), 105-121.

12. Xia, B.; Fang, S.; Chen, X.; Hu, H.; Chen, P.; Wang, H.; Gao, Z., MLKL forms cation channels. Cell Research 2016, 26 (5), 517-528.

13. Yoon, S.; Kovalenko, A.; Bogdanov, K.; Wallach, D., MLKL, the Protein that Mediates Necroptosis, Also Regulates Endosomal Trafficking and Extracellular Vesicle Generation. Immunity 2017, 47 (1), 51-65.e7.

14. Parisi, L. R.; Li, N.; Atilla-Gokcumen, G. E., Very Long Chain Fatty Acids Are Functionally Involved in Necroptosis. Cell Chemical Biology 2017, 24 (12), 1445-1454.e8.

15. Parisi, L. R.; Sowlati-Hashjin, S.; Berhane, I. A.; Galster, S. L.; Carter, K. A.; Lovell, J. F.; Chemler, S. R.; Karttunen, M.; Atilla-Gokcumen, G. E., Membrane Disruption by Very Long Chain Fatty Acids during Necroptosis. ACS Chemical Biology 2019, 14 (10), 2286-2294.

16. Shen, X.; Shen, S.; Li, J.; Hu, Q.; Nie, L.; Tu, C.; Wang, X.; Poulsen, D. J.; Orsburn, B. C.; Wang, J.; Qu, J., lonStar enables high-precision, low-missing-data proteomics quantification in large biological cohorts. Proceedings of the National Academy of Sciences 2018, 115 (21), E4767. 
17. Shen, S.; An, B.; Wang, X.; Hilchey, S. P.; Li, J.; Cao, J.; Tian, Y.; Hu, C.; Jin, L.; Ng, A.; Tu, C.; Qu, M.; Zand, M. S.; Qu, J., Surfactant Cocktail-Aided Extraction/Precipitation/On-Pellet Digestion Strategy Enables Efficient and Reproducible Sample Preparation for Large-Scale Quantitative Proteomics. Analytical Chemistry 2018, 90 (17), 10350-10359.

18. Thinon, E.; Fernandez, J. P.; Molina, H.; Hang, H. C., Selective Enrichment and Direct Analysis of Protein S-Palmitoylation Sites. J Proteome Res 2018, 17 (5), 1907-1922.

19. Jiang, H.; Zhang, X.; Chen, X.; Aramsangtienchai, P.; Tong, Z.; Lin, H., Protein Lipidation: Occurrence, Mechanisms, Biological Functions, and Enabling Technologies. Chemical Reviews 2018, 118 (3), 919-988.

20. Philippe, J. M.; Jenkins, P. M., Spatial organization of palmitoyl acyl transferases governs substrate localization and function. Molecular Membrane Biology 2019, 35 (1), 60-75.

21. Greaves, J.; Munro, K. R.; Davidson, S. C.; Riviere, M.; Wojno, J.; Smith, T. K.; Tomkinson, N. C.; Chamberlain, L. H., Molecular basis of fatty acid selectivity in the zDHHC family of S-acyltransferases revealed by click chemistry. Proc Natl Acad Sci U S A 2017, 114 (8), E1365-e1374.

22. Ohno, Y.; Kihara, A.; Sano, T.; Igarashi, Y., Intracellular localization and tissue-specific distribution of human and yeast DHHC cysteine-rich domain-containing proteins. Biochim Biophys Acta 2006, 1761 (4), 474-83.

23. He, M.; Abdi, K. M.; Bennett, V., Ankyrin-G palmitoylation and betall-spectrin binding to phosphoinositide lipids drive lateral membrane assembly. J Cell Biol 2014, 206 (2), 273-88.

24. Howie, J.; Reilly, L.; Fraser, N. J.; Vlachaki Walker, J. M.; Wypijewski, K. J.; Ashford, M. L.; Calaghan, S. C.; McClafferty, H.; Tian, L.; Shipston, M. J.; Boguslavskyi, A.; Shattock, M. J.; Fuller, W., Substrate recognition by the cell surface palmitoyl transferase DHHC5. Proc Natl Acad Sci U S A 2014, 111 (49), 17534-9.

25. $\quad$ Wang, J.; Hao, J. W.; Wang, X.; Guo, H.; Sun, H. H.; Lai, X. Y.; Liu, L. Y.; Zhu, M.; Wang, H. Y.; Li, Y. F.; Yu, L. Y.; Xie, C.; Wang, H. R.; Mo, W.; Zhou, H. M.; Chen, S.; Liang, G.; Zhao, T. J., DHHC4 and DHHC5 Facilitate Fatty Acid Uptake by Palmitoylating and Targeting CD36 to the Plasma Membrane. Cell Rep 2019, 26 (1), 209-221 e5.

26. Kwiatkowska, K.; Matveichuk, O. V.; Fronk, J.; Ciesielska, A., Flotillins: At the Intersection of Protein S-Palmitoylation and Lipid-Mediated Signaling. Int J Mol Sci 2020, 21 (7). 27. Huang da, W.; Sherman, B. T.; Lempicki, R. A., Bioinformatics enrichment tools: paths toward the comprehensive functional analysis of large gene lists. Nucleic Acids Res 2009, 37 (1), 1-13.

28. Kanehisa, M.; Goto, S., KEGG: kyoto encyclopedia of genes and genomes. Nucleic Acids Res 2000, 28 (1), 27-30.

29. Fan, W.; Guo, J.; Gao, B.; Zhang, W.; Ling, L.; Xu, T.; Pan, C.; Li, L.; Chen, S.; Wang, H.; Zhang, J.; Wang, X., Flotillin-mediated endocytosis and ALIX-syntenin-1-mediated exocytosis protect the cell membrane from damage caused by necroptosis. Science Signaling 2019, 12 (583), eaaw3423.

30. Meister, M.; Tikkanen, R., Endocytic Trafficking of Membrane-Bound Cargo: A Flotillin Point of View. Membranes 2014, 4 (3), 356-371.

31. Gong, Y.-N.; Guy, C.; Olauson, H.; Becker, J. U.; Yang, M.; Fitzgerald, P.; Linkermann, A.; Green, D. R., ESCRT-III Acts Downstream of MLKL to Regulate Necroptotic Cell Death and Its Consequences. Cell 2017, 169 (2), 286-300.e16.

32. Linder, M. E.; Deschenes, R. J., Palmitoylation: policing protein stability and traffic. Nat Rev Mol Cell Biol 2007, 8 (1), 74-84.

33. Fraser, N. J.; Howie, J.; Wypijewski, K. J.; Fuller, W., Therapeutic targeting of protein Sacylation for the treatment of disease. Biochem Soc Trans 2020, 48 (1), 281-290.

34. Ferguson, S. M.; De Camilli, P., Dynamin, a membrane-remodelling GTPase. Nature Reviews Molecular Cell Biology 2012, 13 (2), 75-88. 
35. Macia, E.; Ehrlich, M.; Massol, R.; Boucrot, E.; Brunner, C.; Kirchhausen, T., Dynasore, a Cell-Permeable Inhibitor of Dynamin. Developmental Cell 2006, 10 (6), 839-850.

36. Vandenabeele, P.; Galluzzi, L.; Vanden Berghe, T.; Kroemer, G., Molecular mechanisms of necroptosis: an ordered cellular explosion. Nat Rev Mol Cell Biol 2010, 11 (10), 700-14.

37. Samson, A. L.; Zhang, Y.; Geoghegan, N. D.; Gavin, X. J.; Davies, K. A.; Mlodzianoski, M. J.; Whitehead, L. W.; Frank, D.; Garnish, S. E.; Fitzgibbon, C.; Hempel, A.; Young, S. N.; Jacobsen, A. V.; Cawthorne, W.; Petrie, E. J.; Faux, M. C.; Shield-Artin, K.; Lalaoui, N.; Hildebrand, J. M.; Silke, J.; Rogers, K. L.; Lessene, G.; Hawkins, E. D.; Murphy, J. M., MLKL trafficking and accumulation at the plasma membrane control the kinetics and threshold for necroptosis. Nat Commun 2020, 11 (1), 3151.

38. Murphy, J. M.; Czabotar, P. E.; Hildebrand, J. M.; Lucet, I. S.; Zhang, J. G.; AlvarezDiaz, S.; Lewis, R.; Lalaoui, N.; Metcalf, D.; Webb, A. I.; Young, S. N.; Varghese, L. N.; Tannahill, G. M.; Hatchell, E. C.; Majewski, I. J.; Okamoto, T.; Dobson, R. C.; Hilton, D. J.; Babon, J. J.; Nicola, N. A.; Strasser, A.; Silke, J.; Alexander, W. S., The pseudokinase MLKL mediates necroptosis via a molecular switch mechanism. Immunity 2013, 39 (3), 443-53.

39. Johnston, A.; Wang, Z., Necroptosis: MLKL Polymerization. Journal of Nature and Science (JNSCl); Vol 4, No 72018.

40. Petrie, E. J.; Birkinshaw, R. W.; Koide, A.; Denbaum, E.; Hildebrand, J. M.; Garnish, S. E.; Davies, K. A.; Sandow, J. J.; Samson, A. L.; Gavin, X.; Fitzgibbon, C.; Young, S. N.; Hennessy, P. J.; Smith, P. P. C.; Webb, A. I.; Czabotar, P. E.; Koide, S.; Murphy, J. M., Identification of MLKL membrane translocation as a checkpoint in necroptotic cell death using Monobodies. Proceedings of the National Academy of Sciences 2020, 117 (15), 8468.

41. Blanc, M.; David, F. P. A.; van der Goot, F. G., SwissPalm 2: Protein S-Palmitoylation Database. Methods Mol Biol 2019, 2009, 203-214.

42. Yang, H. Q.; Martinez-Ortiz, W.; Hwang, J.; Fan, X.; Cardozo, T. J.; Coetzee, W. A., Palmitoylation of the KATP channel Kir6.2 subunit promotes channel opening by regulating PIP2 sensitivity. Proc Natl Acad Sci U S A 2020, 117 (19), 10593-10602.

43. Chopard, C.; Tong, P. B. V.; Toth, P.; Schatz, M.; Yezid, H.; Debaisieux, S.; Mettling, C.; Gross, A.; Pugniere, M.; Tu, A.; Strub, J. M.; Mesnard, J. M.; Vitale, N.; Beaumelle, B., Cyclophilin A enables specific HIV-1 Tat palmitoylation and accumulation in uninfected cells. Nat Commun 2018, 9 (1), 2251.

44. Dondelinger, Y.; Declercq, W.; Montessuit, S.; Roelandt, R.; Goncalves, A.; Bruggeman, I.; Hulpiau, P.; Weber, K.; Sehon, C. A.; Marquis, R. W.; Bertin, J.; Gough, P. J.; Savvides, S.; Martinou, J. C.; Bertrand, M. J.; Vandenabeele, P., MLKL compromises plasma membrane integrity by binding to phosphatidylinositol phosphates. Cell Rep 2014, 7 (4), 971-81.

45. Bunney, T. D.; Katan, M., Phosphoinositide signalling in cancer: beyond PI3K and PTEN. Nat Rev Cancer 2010, 10 (5), 342-52.

46. Hammond, G. R. V.; Burke, J. E., Novel roles of phosphoinositides in signaling, lipid transport, and disease. Curr Opin Cell Biol 2020, 63, 57-67.

47. Mucksch, F.; Citir, M.; Luchtenborg, C.; Glass, B.; Traynor-Kaplan, A.; Schultz, C.; Brugger, B.; Krausslich, H. G., Quantification of phosphoinositides reveals strong enrichment of PIP2 in HIV-1 compared to producer cell membranes. Sci Rep 2019, 9 (1), 17661.

48. Sengupta, P.; Lippincott-Schwartz, J., Revisiting Membrane Microdomains and Phase Separation: A Viral Perspective. Viruses 2020, 12 (7).

49. Callan-Jones, A.; Sorre, B.; Bassereau, P., Curvature-driven lipid sorting in biomembranes. Cold Spring Harb Perspect Biol 2011, 3 (2).

50. Monje-Galvan, V.; Klauda, J. B., Modeling Yeast Organelle Membranes and How Lipid Diversity Influences Bilayer Properties. Biochemistry 2015, 54 (45), 6852-6861. 


\section{Supplemental Information for}

\section{Protein acylation by saturated very long chain fatty acids and endocytosis are involved in necroptosis}

Apoorva Pradhan ${ }^{1, \ddagger}$, Daniel Lu ${ }^{1, \ddagger}$, Laura R. Parisi ${ }^{1}$, Shichen Shen ${ }^{2}$, llyas A. Berhane ${ }^{1}$, Samuel L. Galster $^{1}$, Kiana Bynum ${ }^{3}$, Viviana Monje-Galvan ${ }^{4}$, Omer Gokcumen ${ }^{5}$, Sherry R. Chemler ${ }^{1}$, Jun $\mathrm{Qu}^{2}$, Jason $\mathrm{Kay}^{3}, \mathrm{G}$. Ekin Atilla-Gokcumen ${ }^{1^{*}}$

${ }^{1}$ Department of Chemistry, University at Buffalo, The State University of New York, Buffalo, New York 14260, United States

${ }^{2}$ Department of Pharmaceutical Sciences, University at Buffalo, The State University of New York, Buffalo, New York 14214, United States

${ }^{3}$ Department of Oral Biology, University at Buffalo, The State University of New York, Buffalo, New York 14214, United States

${ }^{4}$ Department of Chemical and Biological Engineering, University at Buffalo, The State University of New York, Buffalo, New York 14260, United States

${ }^{5}$ Department of Biological Sciences, University at Buffalo, The State University of New York, Buffalo, New York, 14260, United States

¥These authors contributed equally

*Lead Contact 
Table S1. Results of quantitative proteomics data. Proteins detected in enriched samples from control and necroptotic cells both in IT and OT detection are reported. Related to Figure 2.

This table is provided as a separate spread sheet.

Columns E-J show OT and IT abundances (area under the curve) in control samples (Control 1$3, n=3)$ in log2 scale.

Column K-F show OT and IT abundances (area under the curve) in necroptotic samples (Nec1$3, n=3)$ in $\log 2$ scale

Columns Q-V show OT and IT abundances (area under the curve) in control samples (Control $1-3, n=3$ ) in non-transformed scale.

Columns W-AB show OT and IT abundances (area under the curve) in necroptotic samples (Nec1-3, n=3) in non-transformed scale

Columns $A C$ and $A D$ report that average abundances in control and necroptotic samples in nontransformed scale.

Column AE represents the ratio of protein abundance between necroptotic and control cells.

Column AF reports $p$ values calculated by student's t-test. 
Table S2. Pathways that are enriched in the proteomics data. Related to Figure 5A.

\begin{tabular}{|c|c|c|}
\hline Term & Fold Enrichment & Benjamini \\
\hline hsa03010:Ribosome & 3.3 & $5.42 \mathrm{E}-09$ \\
\hline hsa04130:SNARE interactions in vesicular transport & 4.9 & $6.45 \mathrm{E}-05$ \\
\hline hsa03013:RNA transport & 2.3 & $2.34 \mathrm{E}-04$ \\
\hline hsa04142:Lysosome & 2.6 & 4.16E-04 \\
\hline hsa04730:Long-term depression & 2.8 & 0.03509 \\
\hline hsa04144:Endocytosis & 1.8 & 0.032868 \\
\hline hsa04520:Adherens junction & 2.5 & 0.048754 \\
\hline hsa05130:Pathogenic Escherichia coli infection & 2.8 & 0.048178 \\
\hline hsa04912:GnRH signaling pathway & 2.1 & 0.17522 \\
\hline hsa04145:Phagosome & 1.8 & 0.177291 \\
\hline hsa04141:Protein processing in endoplasmic reticulum & 1.7 & 0.184212 \\
\hline hsa00514:Other types of O-glycan biosynthesis & 3.5 & 0.216658 \\
\hline hsa01200:Carbon metabolism & 1.9 & 0.208579 \\
\hline hsa00970:Aminoacyl-tRNA biosynthesis & 2.2 & 0.225248 \\
\hline hsa01130:Biosynthesis of antibiotics & 1.6 & 0.218812 \\
\hline hsa04961:Endocrine and other factor-regulated calcium reabsorptior & 2.5 & 0.245738 \\
\hline hsa04725:Cholinergic synapse & 1.8 & 0.267471 \\
\hline hsa04530:Tight junction & 1.9 & 0.280981 \\
\hline hsa04611:Platelet activation & 1.7 & 0.272356 \\
\hline hsa04540:Gap junction & 1.9 & 0.277794 \\
\hline hsa05203:Viral carcinogenesis & 1.5 & 0.291096 \\
\hline hsa00510:N-Glycan biosynthesis & 2.3 & 0.293641 \\
\hline hsa04720:Long-term potentiation & 2.0 & 0.310928 \\
\hline hsa04727:GABAergic synapse & 1.8 & 0.355146 \\
\hline hsa00604:Glycosphingolipid biosynthesis - ganglio series & 3.7 & 0.349585 \\
\hline hsa00533:Glycosaminoglycan biosynthesis - keratan sulfate & 3.7 & 0.349585 \\
\hline hsa03050:Proteasome & 2.3 & 0.339607 \\
\hline hsa04977:Vitamin digestion and absorption & 3.0 & 0.344636 \\
\hline hsa05100:Bacterial invasion of epithelial cells & 1.9 & 0.349461 \\
\hline hsa05169:Epstein-Barr virus infection & 1.6 & 0.34072 \\
\hline hsa04921:Oxytocin signaling pathway & 1.6 & 0.34369 \\
\hline hsa00020:Citrate cycle (TCA cycle) & 2.6 & 0.34054 \\
\hline hsa04724:Glutamatergic synapse & 1.7 & 0.332245 \\
\hline hsa04915:Estrogen signaling pathway & 1.7 & 0.381762 \\
\hline hsa00534:Glycosaminoglycan biosynthesis - heparan sulfate / hepa & 2.8 & 0.375933 \\
\hline hsa03015:mRNA surveillance pathway & 1.7 & 0.384226 \\
\hline hsa05416:Viral myocarditis & 2.0 & 0.395866 \\
\hline hsa04666:Fc gamma R-mediated phagocytosis & 1.7 & 0.410686 \\
\hline hsa00601:Glycosphingolipid biosynthesis - lacto and neolacto series & 2.6 & 0.434436 \\
\hline hsa00564:Glycerophospholipid metabolism & 1.6 & 0.439491 \\
\hline hsa04713:Circadian entrainment & 1.6 & 0.439491 \\
\hline hsa05205:Proteoglycans in cancer & 1.4 & 0.449018 \\
\hline hsa04340:Hedgehog signaling pathway & 2.5 & 0.454656 \\
\hline hsa04670:Leukocyte transendothelial migration & 1.5 & 0.448045 \\
\hline hsa02010:ABC transporters & 2.0 & 0.461686 \\
\hline hsa04962:Vasopressin-regulated water reabsorption & 2.0 & 0.461686 \\
\hline
\end{tabular}


Figure S1. Related to Figure 1. (A) Relative fluorescence intensity of pMLKL immunostaining in control and necroptotic cells. A minimum of 30 cells per condition were measured, $n=3$, *** represents $P$ value $<0.001$ by two-tailed unpaired $t$ test. (B)The scatterplots of $\log _{2}$ Mean IT and OT intensities shown in $x$ and $y$ axes, respectively. Each dot represents an individual protein detected in control (blue) and necroptotic cells (red).
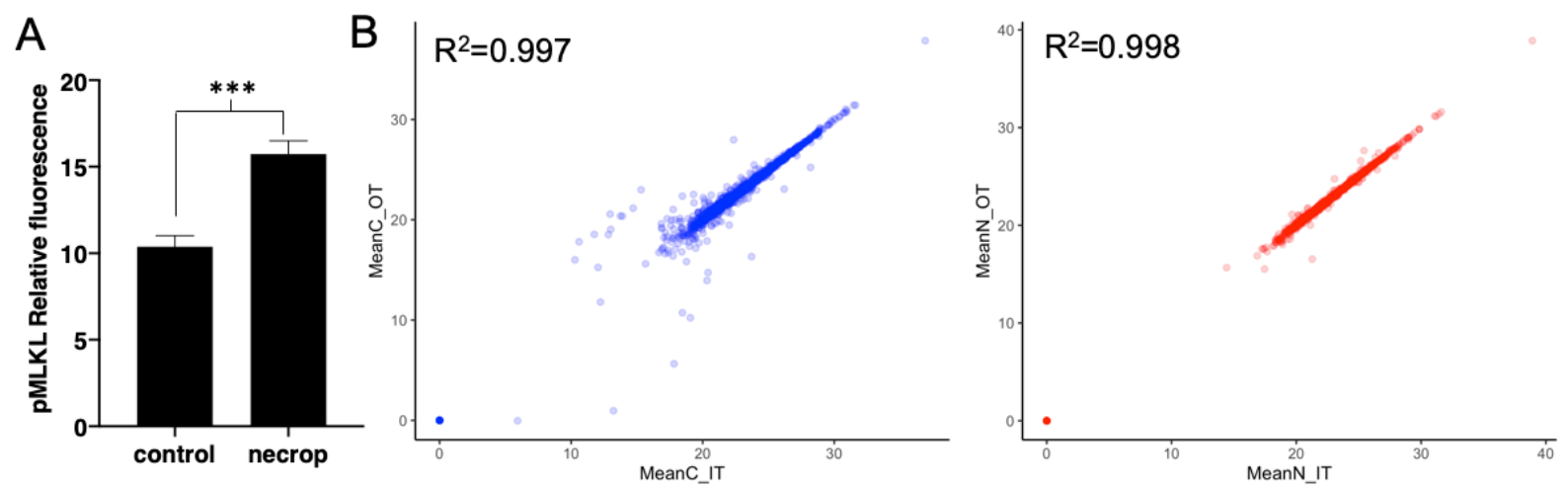
Figure S2. Violin plot of log2 mean intensities of proteins (y axis) detected in individual experimental categories ( $x$ axis). Related to Figure 2A-B. The width of each violin plot corresponds to the number of observations at a given log2 mean intensity. Note that the intensities show a bimodal distribution where proteins are either show intensities range 20-30 or close to zero. Also note that the proportion of proteins that show intensities close to zero in the control group is less as compared to necroptosis group.

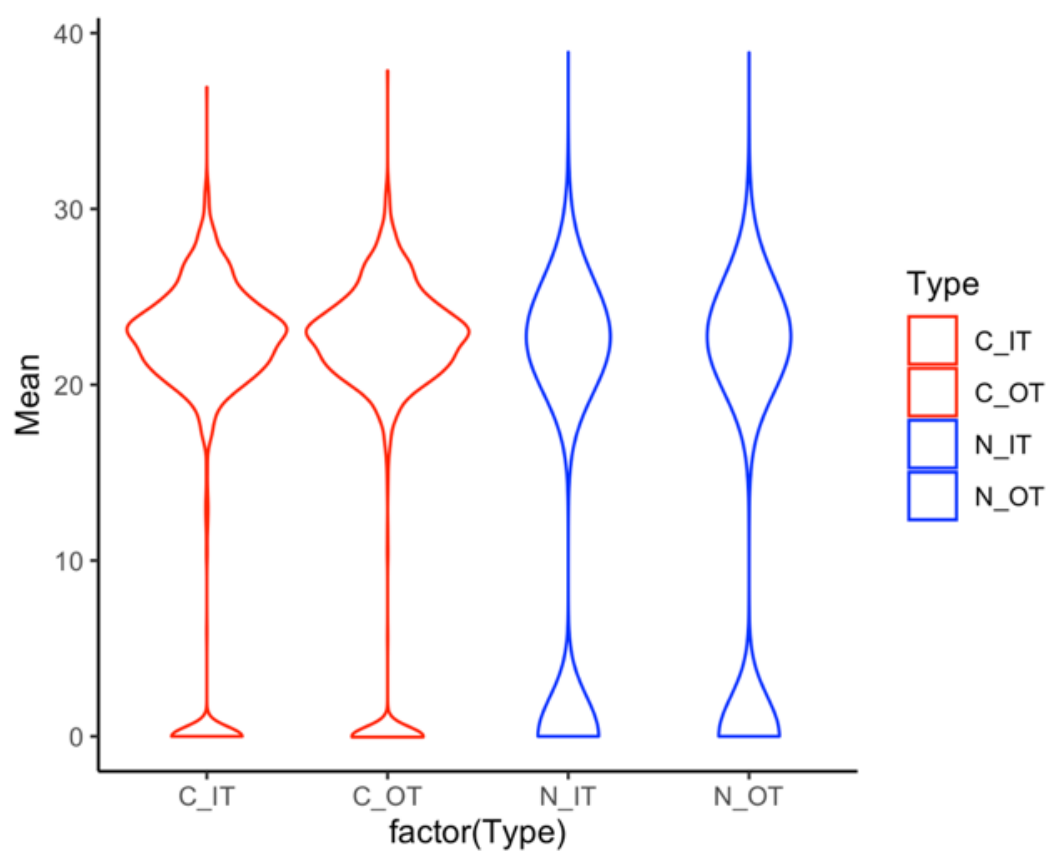


Figure S3. Related to Figure 3. (A) Levels of MLKL and pMLKL in NSA treated necroptotic cells. Necroptosis is induced with BV6/zVAD-FMK/TNF- $\alpha$ treatment. Western blot analysis of the whole lysates of NSA treated control and necroptotic cells show that NSA treatment does not affect cellular levels of MLKL and pMLKL during necroptosis. The proteins on the beads were blotted for MLKL and pMLKL with a-tubulin as the loading control. (B) Acylation of MLKL and pMLKL. Western blot analysis of proteins from C16:0 alkFA treated control and necroptosis cells. After cell lysis and Biotin-azide attachment via CuAAC, acylated proteins are enriched on neutravidin resin. The samples are blotted for MLKL and pMLKL and Calnexin. Calnexin is used as a loading control.
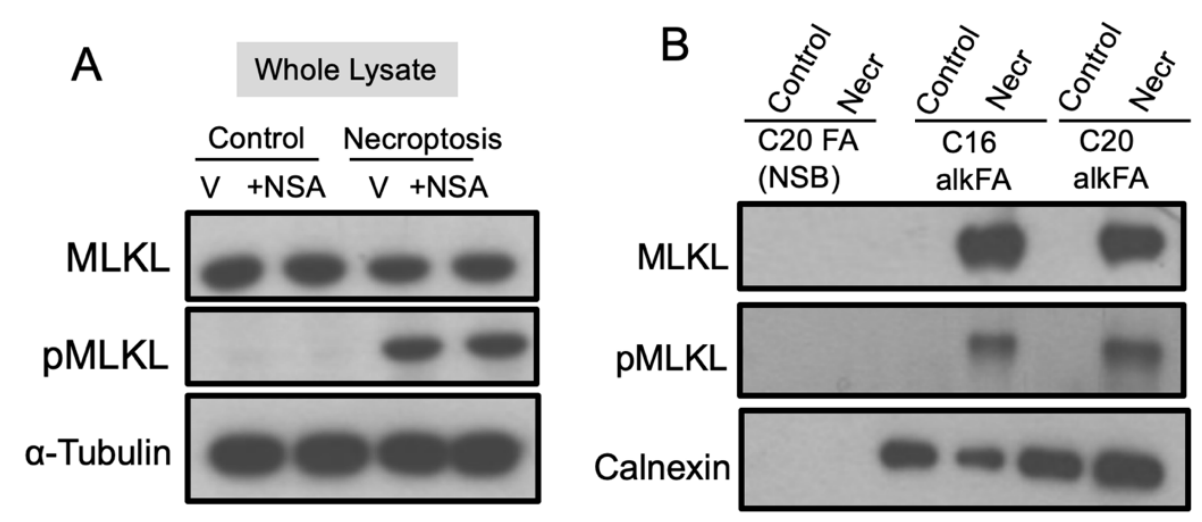


\section{Materials and Methods.}

HT-29 (human colorectal adenocarcinoma epithelial) cells were obtained from the American Type Culture Collection. Dulbecco's Modification of Eagle's Medium (DMEM), penicillin-streptomycin and trypsin were acquired from Corning. Fetal Bovine Serum (FBS) and dimethyl sulfoxide (DMSO) (Cat \#42780) were obtained from Sigma-Aldrich and Alfa Aesar, respectively. Antibodies were obtained from Abcam (rabbit monoclonal anti-phospho S358-MLKL, Cat. \#ab187091; rabbit polyclonal anti-calnexin, Cat. \#ab22595), Cell Signaling Technology (rabbit monoclonal antiMLKL, Cat. \#14993; rabbit monoclonal anti-calnexin, Cat. \#2679S), Promega (goat anti-rabbit HRP conjugate, Cat. \# W4011), and Jackson Immunoresearch Lab (goat anti-mouse HRP conjugate, Cat. \#115-035-174).

BV6 was obtained from Selleck Chemicals (Cat \#S7597), zVAD-FMK was obtained from Enzo (Cat \#ALX-260-020), TNF- $\alpha$ was obtained from R\&D Systems (Cat \#210-TA/CF). Dynasore (Cat. \# 14062) and TBTA were obtained from Cayman Chemical. Protease inhibitor (Cat \#A32955) and high capacity neutravidin-agarose resin (Cat. \# 29202) were obtained from Thermo Fisher Scientific. TCEP hydrochloride (Cat \#K831) was obtained from VWR AMRESCO and fatty acidfree BSA (Cat \#A7030) was obtained from Millipore Sigma. MTT reagent (Cat \#L11939) was obtained from Alfa Aesar. For synthesis of C20 alkFA, 1- bromoheptadecane was obtained from TCI America; all other reagents were obtained from Millipore Sigma. The synthesis of C20:0 alkFA was carried out as described previously. ${ }^{1}$

Cell culture. HT-29 cells were cultured at $37{ }^{\circ} \mathrm{C}$ in $5 \% \mathrm{CO} 2$ atmosphere in DMEM supplemented with $10 \%(\mathrm{v} / \mathrm{v})$ fetal bovine serum and $1 \%(\mathrm{v} / \mathrm{v})$ penicillin/streptomycin solution. Cells were cultured for approximately 2 months and were routinely checked for mycoplasma infection. Cells were plated according to the requirement of each experiment as described below.

Necroptosis treatment. To induce necroptosis cell death, HT-29 cells were initially sensitized to TNF-dependent cell death pathway by SMAC mimetic BV6 $(1 \mu \mathrm{M})$, and were co-treated with pan-caspase inhibitor zVAD-FMK $(25 \mu \mathrm{M})$ and incubated for $30 \mathrm{~min}$ at $37^{\circ} \mathrm{C}$. Cells were then treated with TNF- $\alpha(10 \mathrm{ng} / \mathrm{mL})$ and was incubated for $3 \mathrm{~h}$.

Apoptosis treatment. Cells were sensitized to TNF-dependent apoptosis cell death by using SMAC mimetic BV6. HT-29 cells were treated with BV6 $(1 \mu \mathrm{M})$ for $30 \mathrm{~min}$. TNF-alpha (10 $\mathrm{ng} / \mathrm{mL}$ ) was added after the 30 min BV6 treatment to initiate apoptosis for $24 \mathrm{~h}$.

Cell Viability Assays. HT-29 cells were seeded 96-well plates with 30,000 cells per well for 20 $\mathrm{h}$ before pretreatment with small molecule inhibitors. After the designated pretreatment time and/or induction of cell death, the 96 -well plate was centrifuged for $2 \mathrm{~min}$ at $200 \mathrm{rcf}$ at room 
temperature. The media was removed from each well and replaced with $200 \mu \mathrm{L}$ of fresh media that contains $0.5 \mathrm{mg} / \mathrm{mL}$ of MTT reagent. The plate was incubated at $37^{\circ} \mathrm{C}$ for $2.5 \mathrm{~h}$ and then centrifuged for 2 min at room temperature. $155 \mu \mathrm{L}$ of media was removed from each well and added $90 \mu \mathrm{L}$ of DMSO was added back. The plate was then incubated at $37^{\circ} \mathrm{C}$ for $10 \mathrm{~min}$. Each well was then re-suspended to solubilize the formazan crystal and was centrifuged at 1000 rcf for $2 \mathrm{~min}$ at room temperature. Absorbance was measured at $550 \mathrm{~nm}$ using Biotek Synergy $\mathrm{H} 1$ plate reader. In order to calculate the percent viability of treated cells compared to control cells, the raw absorbance values of cells were subtracted with the average absorbance values of blanks $(n \geq 4)$. All corrected absorbance values were normalized to the average absorbance values of vehicle control cells and was expressed as percentage cell viability $(n \geq 4)$. Results are representative of at least two independent experiments

C20 alkFA delivery and protein enrichment. C20 alkFA was prepared as described previously. ${ }^{1} \mathrm{HT}-29$ cells $\left(1 \times 10^{7}\right)$ were plated in $10 \mathrm{~cm}$ dishes for $\sim 16 \mathrm{~h}$. 30 dishes were used so that there were enough to combine 2 plates per sample for control, 4 plates per sample for necroptosis for 4 replicates per condition treated with alkFA, and one replicate per condition treated with FA to assess non-specific binding (NSB). Cells were starved for $2 \mathrm{~h}$ and treated to induce necroptosis (final concentrations of $1 \mu \mathrm{M} B V 6,25 \mu \mathrm{M} z \mathrm{VAD}, 10 \mathrm{ng} / \mathrm{mL}$ TNF) then (alk)FA was added for $3 \mathrm{~h}$ before collection. Treatments were staggered to facilitate cell collection:

The complete media was removed and replaced with $7 \mathrm{~mL}$ DMEM with no additives. 20 $\mathrm{mM}$ arachidic acid (C20 FA) and $\omega$-alkynyl C20 fatty acid (C20 alkFA) were prepared in ethanol. C20 alkFA was diluted to $0.4 \mathrm{mM}$ in pre-warmed sterile-filtered $10 \%$ fatty acid-free BSA in DMEM with no additives and sonicated at $50^{\circ} \mathrm{C}$ until use ( 1-2 h). C20 FA (2.5 mg) was dissolved in $400 \mu \mathrm{L}$ ethanol, and $160 \mu \mathrm{L}$ was transferred to $8 \mathrm{~mL} 10 \%$ FA-free BSA. C20 alkFA (3.4 mg) was dissolved in $550 \mu \mathrm{L}$ ethanol and transferred to $27.5 \mathrm{~mL}$ FA-free BSA. After $90 \mathrm{~min}$ starvation, DMSO was added to control flasks or BV6 and zVAD. After 30 min, TNF-alpha was added to necroptosis followed by $1 \mathrm{~mL}$ of alkFA solution. Plates were gently rocked to further mix and incubated for $3 \mathrm{~h}$ before collection.

Cells were scraped from the plates on ice and transferred to $50 \mathrm{~mL}$ centrifuge tubes, combining 2 plates per sample for control cells, and 4 plates per sample for necroptotic cells. Plates were rinsed with $4 \mathrm{~mL}$ PBS after scraping, which was also transferred to the centrifuge tube. Cells were centrifuged for $5 \mathrm{~min}$ at $500 \mathrm{rcf}$ at $4{ }^{\circ} \mathrm{C}$. The supernatant was decanted and the cell pellets were resuspended with $3 \mathrm{~mL}$ PBS. An additional $7 \mathrm{~mL}$ PBS was added and the cells were centrifuged again for $5 \mathrm{~min}$ at $500 \mathrm{rcf}$ at $4{ }^{\circ} \mathrm{C}$. The supernatant was decanted and $1050 \mu \mathrm{L}$ 
PBS was added and the cells were resuspended, from which $50 \mu \mathrm{L}$ was transferred to an Eppendorf tube for protein measurements using Bradford assay, and the remainder was stored at $-80{ }^{\circ} \mathrm{C}$.

Samples were thawed on ice, resuspended in PBS with protease inhibitor with volumes normalized based on protein concentration, then sonicated on a cold block, mixed and transferred into $1 \mathrm{~mL}$ ultracentrifuge tube containing $5 \mathrm{~mL}$ of PBS with protease inhibitor. Samples were centrifuged for $1 \mathrm{~h}$ at $25,000 \mathrm{rpm}$ at $4{ }^{\circ} \mathrm{C}$. The supernatant was decanted and $250 \mu \mathrm{L} 4 \%$ SDS in PBS was added to the cell pellets, which were partially resuspended using a pipette and transferred to an Eppendorf tube, then sonicated. They were placed on ice and 250 $\mu \mathrm{L}$ PBS was added. Once chilled, $500 \mu \mathrm{L}$ ice-cold methanol and $200 \mu \mathrm{L}$ ice-cold chloroform were added. The tubes were vortexed then centrifuged for $10 \mathrm{~min}$ at maximum speed $(16,900$ rcf) at $4{ }^{\circ} \mathrm{C}$. The liquid layers were carefully decanted and $500 \mu \mathrm{L}$ methanol was added to the pellet, which was then sonicated and centrifuged for 5 min at maximum speed at $4{ }^{\circ} \mathrm{C}$. The supernatant was decanted and the pellets were resuspended in $488 \mu \mathrm{L} 4 \%$ SDS in PBS with sonication.

A mastermix was prepared for the CuAAC reaction using $204 \mu \mathrm{L}$ of $25 \mathrm{mM} \mathrm{CuSO}_{4}, 510$ $\mu \mathrm{L}$ of $2.5 \mathrm{mM}$ TBTA, $136 \mu \mathrm{L}$ of $9.4 \mathrm{mM}$ biotin-PEG -azide, and $102 \mu \mathrm{L} 50 \mathrm{mM}$ neutralized TCEP. TCEP was added last to the mastermix, which was then immediately vortexed and $112 \mu \mathrm{L}$ were added to each sample. The samples were vortexed and incubated at $37^{\circ} \mathrm{C}$ for $1 \mathrm{~h}$.

After the CuAAC reaction, the tubes were placed on ice and allowed to chill before 600 $\mu \mathrm{L}$ ice-cold methanol and $240 \mu \mathrm{L}$ ice-cold chloroform were added. Samples were vortexed then centrifuged for $10 \mathrm{~min}$ at maximum speed at $4{ }^{\circ} \mathrm{C}$. The liquid layers were carefully decanted and $500 \mu \mathrm{L}$ methanol was added to the pellet, which was then sonicated and centrifuged for $5 \mathrm{~min}$ at maximum speed at $4{ }^{\circ} \mathrm{C}$. The supernatant was decanted and another $500 \mu \mathrm{L}$ methanol was added to the pellet, which was then sonicated and centrifuged for $5 \mathrm{~min}$ at maximum speed at 4 ${ }^{\circ} \mathrm{C}$. The pellets were then dissolved by sonication in $325 \mu \mathrm{L} 2 \%$ SDS in PBS. Aliquots were taken for protein measurement using BCA assay.

The samples were normalized according to protein concentration and $300 \mu \mathrm{L}$ taken into a new Eppendorf tube for enrichment. High capacity neutravidin-agarose resin was resuspended and for each sample $40 \mu \mathrm{L}$ slurry was transferred into a $15 \mathrm{~mL}$ centrifuge tube containing $10 \mathrm{~mL} 0.2 \%$ SDS in PBS. This was centrifuged for $2 \mathrm{~min}$ at $2000 \mathrm{rcf}$ at room temperature, then the supernatant was carefully removed with suction. Another $10 \mathrm{~mL} 0.2 \%$ SDS in PBS was added to the resin and the tubes were capped and inverted several times to mix, then centrifuged again for 2 min at 2000 rcf at room temperature. The supernatant was 
removed and a third wash with $0.2 \%$ SDS in PBS was similarly performed. The resin was resuspended in $150 \mu \mathrm{L} 1 \%$ Brij-97 in PBS then transferred to the sample. The samples were rotated end-over-end for $90 \mathrm{~min}$ at room temperature, then centrifuged for $2 \mathrm{~min}$ at $2000 \mathrm{rcf}$ at room temperature. The resin was transferred to a $15 \mathrm{~mL}$ centrifuge tube containing $9 \mathrm{~mL} 0.2 \%$ SDS in PBS using two $500 \mu \mathrm{L}$ portions of $0.2 \%$ SDS in PBS. The samples were centrifuged for $2 \mathrm{~min}$ at $2000 \mathrm{rcf}$ at room temperature, then the supernatant was carefully removed with suction. Samples were washed with $10 \mathrm{~mL} 0.2 \%$ SDS in PBS twice more. An additional three washes with PBS only were performed, then the supernatant was carefully removed and the resin was stored at $-80{ }^{\circ} \mathrm{C}$ for proteomics analysis.

\section{Quantitative proteomics}

Protein digestion. A surfactant-aided precipitation/on-bead digestion method was modified from our previous sample preparation protocol ${ }^{2}$ and employed in the current study. In brief, beads were re-suspended with $60 \mathrm{uL} 0.5 \%$ SDS, and were sequentially incubated with $10 \mathrm{mM}$ dithiothreitol (DTT) at $56{ }^{\circ} \mathrm{C}$ for $30 \mathrm{~min}$ and $25 \mathrm{mM}$ iodoacetamide (IAM) at $37^{\circ} \mathrm{C}$ for 30 min to denature protein and dissociate protein disulfide bonds. Both steps were conducted with rigorous oscillation in darkness using a thermomixer (Eppendorf). A total of 7 volumes of chilled acetone was then added to the beads with constant vortexing, and the mixture was incubated at $-20{ }^{\circ} \mathrm{C}$ for $3 \mathrm{hr}$. After centrifugation at $20,000 \mathrm{~g}, 4{ }^{\circ} \mathrm{C}$ for $30 \mathrm{~min}$, liquid was removed and the beads was rinsed by $500 \mathrm{uL}$ methanol and left to air-dry. A volume of $55 \mathrm{uL} 50 \mathrm{mM} \mathrm{pH} 8.4$ Trisformic acid (FA) was then added to the beads, and a total volume of $5 \mathrm{uL}$ trypsin (Sigma Aldrich) dissolved in $50 \mathrm{mM} \mathrm{pH} 8.4$ Tris-FA was added for 6-hr digestion at $37^{\circ} \mathrm{C}$ with rigorous oscillation in a thermomixer. Digestion was terminated by addition of $0.6 \mathrm{uL} \mathrm{FA}$, and beads were pelleted by centrifugation at $20,000 \mathrm{~g}, 4^{\circ} \mathrm{C}$ for $30 \mathrm{~min}$. Supernatant was carefully transferred to $\mathrm{LC}$ vials for analysis.

LC-MS analysis. The LC-MS system consists of a Dionex Ultimate 3000 nano LC system, a Dinex Ultimate 3000 micro LC system with a WPS-3000 autosampler, and an Orbitrap Fusion Lumos mass spectrometer. A large-inner diameter (i.d.) trapping column (300-um i.d. $x 5 \mathrm{~mm}$ ) was implemented before the nano LC column (75-um i.d. x $65 \mathrm{~cm}$, packed with 2.5-um Xselect CSH C18 material) for high-capacity sample loading, cleanup and delivery. For each sample, 4 $\mathrm{UL}$ derived peptides was injected twice consecutively for LC-MS analysis. Mobile phase $A$ and $B$ were $0.1 \% \mathrm{FA}$ in $2 \%$ acetonitrile (ACN) and $0.1 \% \mathrm{FA}$ in $88 \% \mathrm{ACN}$. The 180-min LC gradient profile was: $4 \%$ for $3 \mathrm{~min}, 4-11$ for $5 \mathrm{~min}, 11-32 \%$ B for $117 \mathrm{~min}, 32-50 \%$ B for $10 \mathrm{~min}, 50-97 \%$ B for $5 \mathrm{~min}, 97 \%$ B for $7 \mathrm{~min}$, and then equilibrated to $4 \%$ for $27 \mathrm{~min}$. The mass spectrometer was 
operated under data-dependent acquisition (DDA) mode with a maximal duty cycle of $3 \mathrm{~s}$. MS1 spectra was acquired by Orbitrap (OT) under 120k resolution for ions within the $\mathrm{m} / \mathrm{z}$ range of 400 1,500. Automatic Gain Control (AGC) and maximal injection time was set at $120 \%$ and $50 \mathrm{~ms}$, and dynamic exclusion was set at $45 \mathrm{~s}, \pm 10 \mathrm{ppm}$. Precursor ions were isolated by quadrupole using a m/z window of $1.2 \mathrm{Th}$, and were fragmented by high-energy collision dissociation (HCD). MS2 spectra was acquired differently in the two LC-MS injections. One was by OT under 15k resolution with a maximal injection time of $50 \mathrm{~ms}$, and the other was by lon trap (IT) under Rapid scan rate with a maximal injection time of $50 \mathrm{~ms}$. Detailed LC-MS settings and relevant information are enclosed in a previous publication by Shen et al. ${ }^{3}$

Data processing. LC-MS files were searched against Homo Sapiens Swiss-Prot protein sequence database (20,350 entries) using Sequest HT embedded in Proteome Discoverer 1.4 (Thermo Fisher Scientific). Target-decoy searching approach using a concatenated forward and reverse protein sequence database was applied for FDR estimation purposes. Searching parameters include: 1) Precursor ion mass tolerance: 20 ppm; 2) Product ion mass tolerance: 0.02 Da for OT, 0.8 Da for IT; 3) Maximal missed cleavages per peptide: 2; 4) Fixed modifications: carbamidomethylation of cysteine; 5) Dynamic modifications: Oxidation of methionine, Acetylation of peptide $\mathrm{N}$-terminals. Peptide filtering, protein inference and grouping, and FDR control were accomplished by Scaffold v4.8.4 (Proteome Software, Inc.) The filtered peptide-spectrum match (PSM) list was exported. Proteomic quantification was performed by an in-house developed MS1-based quantitative method, IonStar to achieve high-precision, lowmissing data quantification. ${ }^{4}$ IonStar involves SIEVE v2.2 (ThermoFisher Scientific) and an inhouse developed $R$ package lonStarStat. The major procedures include: i) Chromatographic alignment with ChromAlign for inter-run calibration of retention time (RT) shift. Quality control and selection of the optimal reference for alignment were accomplished by monitoring alignment scores (>0.8) and base-peak ion chromatogram intensity; ii) Data-independent MS1 quantitative feature generation using the DICE method, which extracts ion chromatograms for all precursor ions with corresponding MS2 scans in the aligned dataset with a defined m/z-RT window (10 ppm, $1 \mathrm{~min}$ ). iii) Integration of the SQL database exported from SIEVE (containing corresponding intensities of all quantitative features in the sample set) and the filtered PSM list by MS2 scan number using a customized R script. Frames with valid PSMs were then subjected to dataset-wide normalization, multivariate outlier detection/removal, and aggregation to protein level using lonStarStat. Detailed steps of the lonStar method can be found in the user manual (available at https://github.com/shichens1989). 


\section{Microscopy}

HT-29 cells were seeded onto glass coverslips in 24-well plates and treated as above to induce necroptosis. Following fixation with $4 \%$ paraformaldehyde, the cells were permeabilized and immunostained with rabbit monoclonal anti-phospho S358-MLKL, (Cell Signaling, Cat.

\#91689S) followed by Alexa-488 goat anti-rabbit secondary, (Jackson ImmunoResearch, Cat. \#111-545-144) along with the nuclear DNA stain DAPI. ${ }^{5}$ After mounting on slides, microscopy was performed on a Dragonfly spinning disk confocal (Andor) and analyzed with $\mathrm{FIJI}^{6}$ to determine the average pMLKL fluorescence intensity.

1. Parisi, L. R.; Sowlati-Hashjin, S.; Berhane, I. A.; Galster, S. L.; Carter, K. A.; Lovell, J. F.; Chemler, S. R.; Karttunen, M.; Atilla-Gokcumen, G. E., Membrane Disruption by Very Long Chain Fatty Acids during Necroptosis. ACS Chemical Biology 2019, 14 (10), 2286-2294.

2. Shen, S.; An, B.; Wang, X.; Hilchey, S. P.; Li, J.; Cao, J.; Tian, Y.; Hu, C.; Jin, L.; Ng, A.; Tu, C.; Qu, M.; Zand, M. S.; Qu, J., Surfactant Cocktail-Aided Extraction/Precipitation/On-Pellet Digestion Strategy Enables Efficient and Reproducible Sample Preparation for Large-Scale Quantitative Proteomics. Analytical Chemistry 2018, 90 (17), 10350-10359.

3. Shen, X.; Shen, S.; Li, J.; Hu, Q.; Nie, L.; Tu, C.; Wang, X.; Orsburn, B.; Wang, J.; Qu, J., An lonStar Experimental Strategy for MS1 Ion Current-Based Quantification Using UltrahighField Orbitrap: Reproducible, In-Depth, and Accurate Protein Measurement in Large Cohorts. Journal of Proteome Research 2017, 16 (7), 2445-2456.

4. Shen, X.; Shen, S.; Li, J.; Hu, Q.; Nie, L.; Tu, C.; Wang, X.; Poulsen, D. J.; Orsburn, B. C.; Wang, J.; Qu, J., lonStar enables high-precision, low-missing-data proteomics quantification in large biological cohorts. Proceedings of the National Academy of Sciences 2018, 115 (21), E4767.

5. $\quad$ Croft, A. J.; Metcalfe, S.; Honma, K.; Kay, J. G., Macrophage Polarization Alters Postphagocytosis Survivability of the Commensal Streptococcus gordonii. Infect Immun 2018, 86 (3).

6. Schindelin, J.; Arganda-Carreras, I.; Frise, E.; Kaynig, V.; Longair, M.; Pietzsch, T.; Preibisch, S.; Rueden, C.; Saalfeld, S.; Schmid, B.; Tinevez, J. Y.; White, D. J.; Hartenstein, V.; Eliceiri, K.; Tomancak, P.; Cardona, A., Fiji: an open-source platform for biological-image analysis. Nat Methods 2012, 9 (7), 676-82. 